\title{
Revisiting the relation between momentum and scalar roughness lengths of urban surfaces
}

Article

Accepted Version

Li, Q., Bou-Zeid, E., Grimmond, S., Zilitinkevich, S. and Katul, G. (2020) Revisiting the relation between momentum and scalar roughness lengths of urban surfaces. Quarterly Journal of the Royal Meteorological Society, 146 (732). pp. 3144-3164. ISSN 1477-870X doi: https://doi.org/10.1002/qj.3839 Available at https://centaur.reading.ac.uk/91220/

It is advisable to refer to the publisher's version if you intend to cite from the work. See Guidance on citing.

Published version at: http://dx.doi.org/10.1002/aj.3839

To link to this article DOI: http://dx.doi.org/10.1002/qj.3839

Publisher: Wiley

All outputs in CentAUR are protected by Intellectual Property Rights law, including copyright law. Copyright and IPR is retained by the creators or other copyright holders. Terms and conditions for use of this material are defined in the End User Agreement.

www.reading.ac.uk/centaur 
Central Archive at the University of Reading

Reading's research outputs online 
2 Journal Section

\title{
Revisiting the Relation Between Momentum and Scalar Roughness Lengths of Urban Surfaces
}

\author{
Qi Li ${ }^{1 * 2}$ | Elie Bou-Zeid ${ }^{2}$ | Sue Grimmond ${ }^{3}$ | Sergej
}

Zilitinkevich $^{4}$ | Gabriel Katul ${ }^{5,6,2}$

${ }^{1}$ School of Civil and Environmental Engineering, Ithaca, NY, USA

$6{ }^{2}$ Department of Civil and Environmental Engineering, Princeton, NJ, USA

${ }^{3}$ Department of Meteorology, University of Reading, Reading, UK

${ }^{4}$ Finnish Meteorological Institute, P.O. Box 503, 00101 Helsinki, Finland

${ }^{5}$ Nicholas School of the Environment, Duke University, Durham, NC, USA

${ }^{6}$ Department of Civil and Environmental Engineering, Duke University, Durham, NC, USA

\section{Correspondence}

Email: q156@cornell.edu

Funding information

Support was provided by start-up funds from Cornell University; the small allocation grant for new faculty by the National Center for Atmospheric Research (NCAR), NOAA-Princeton Cooperative Institute for Climate Science (CU00029), Princeton's Andlinger Center for Energy and the Environment, and the U.S. National Science Foundation (NSF) Sustainability Research Network (Cooperative Agreement 1444758 and grant 1664091). High-performance computing support from Cheyenne (doi:10.5065/D6RX99HX) provided by NCAR's Computational and Information Systems Laboratory (NSF projects P36861020 and UPRI0007) is acknowledged. SZ acknowledges support from the Academy of Finland (grant 314 798/799), and GK acknowledges partial support from NSF-AGS-1644382 and NSF-IOS-1754893. The Metropolis Project is acknowledged for supporting the collaborative visit of GK to Princeton University.

\author{
Large Eddy Simulations (LES) of neutral flow over regular
}


arrays of cuboids are conducted to explore connections between momentum $\left(z_{0 m}\right)$ and scalar $\left(z_{0 s}\right)$ roughness lengths in urban environments, and how they are influenced by surface geometry. As LES resolves the obstacles but not the micro-scale boundary layers attached to them, the aforementioned roughness lengths are analyzed at two distinct spatial scales. At the micro-scale (roughness of individual facets, e.g. roofs), it is assumed that both momentum and scalar transfer are governed by accepted arguments for smooth walls that form the basis for the LES wall model. At the macro-scale, the roughness lengths are representative of the aggregate effects of momentum and scalar transfer over the resolved roughness elements of the whole surface, and hence they are directly computed from the LES. The results indicate that morphologically-based parameterizations for macro-scale $z_{0 m}$ are adequate overall. The relation between the momentum and scalar macro-roughness values, as conventionally represented by $\log \left(z_{0 m} / z_{0 s}\right)$ and assumed to scale with $R e_{*}^{n}$ (where $R e_{*}$ is a roughness Reynolds number), is then interpreted using surface renewal theory (SRT). SRT predicts $n=1 / 4$ when only Kolmogorov-scale eddies dominate the scalar exchange, whereas $n=1 / 2$ is predicted when large eddies limit the renewal dynamics. The latter is found to better capture the LES results. However, both scaling relations indicate that $z_{0 s}$ decreases when $z_{0 m}$ increases for typical urban geometries and scales. This is opposite to how their relation is usually modeled for urban canopies (i.e. $z_{0 s} / z_{0 m}$ is a fixed value smaller than unity).

\section{KEYWORDS}

large-eddy simulation, scalar roughness length, surface renewal theory, urban canopy

\section{$\therefore 1$ | INTRODUCTION}

\section{- 1.1 | Overview of roughness lengths}

Quantification of momentum, heat, water vapor and other trace gas fluxes over built surfaces remains a first-order 
Bou-Zeid et al., 2009; Llaguno-Munitxa and Bou-Zeid, 2018). Monin-Obukhov similarity theory (MOST) (Monin and Obukhov, 1954) remains the cornerstone for representing these turbulent exchanges between the earth surface and the lower atmosphere although possible refinements accounting for deviations from MOST in the roughness sublayer have been proposed for urban (Theeuwes et al., 2019) and vegetated canopies (Garratt, 1980; Harman and Finnigan, 2007, 2008). The roughness lengths used in MOST are of significance in coupled urban-atmospheric models: they encapsulate how turbulent exchanges depend on the characteristics of the underlying surface such as geometric or thermal properties, and on the spatial distributions of scalar sources/sinks at the surface (Garratt, 1992). In MOST, the momentum and scalar roughness lengths $\left(z_{0 m}\right.$ and $\left.z_{0 s}\right)$ for neutral atmospheric flows are used to determine the surface drag (represented by the friction velocity $u_{*}$ ) and scalar fluxes (represented by the scalar, here temperature, scale $\theta_{*}$ ) based on the mean profiles of streamwise velocity $\bar{u}$ and temperature $\bar{\theta}$ via

$$
u_{*}=\bar{u}\left[\frac{1}{\kappa} \log \left(\frac{z-d}{z_{0 m}}\right)\right]^{-1}
$$

and

$$
\theta_{*}=\left(\bar{\theta}_{s}-\bar{\theta}\right)\left[\frac{1}{\kappa} \log \left(\frac{z-d_{s}}{z_{0 s}}\right)\right]^{-1},
$$

where $\kappa$ is the von Kármán constant ( $\kappa$ is assumed to be 0.4 ); $d$ and $d_{s}$ are the displacement heights of momentum and scalars, respectively; $\bar{\theta}_{s}$ is the 'aerodynamic' mean surface temperature, which is obtained by extending the scalar logarithmic profile to $z=z_{0 s}+d_{s} ; u_{*}$ is the friction velocity; and $\theta_{*}=F_{c} / u_{*}$ where $F_{c}$ is the kinematic surface scalar flux (here sensible heat flux).

Roughness lengths and corollary concepts such as friction factor have been extensively studied in both the engineering and the atmospheric sciences communities since the early 20th century. Rough-wall turbulent boundary layers were examined in the classic experiments by Nikuradse (1933) for turbulent flows over sand roughness covering the walls of a pipe in the absence of buoyancy forces. Since then, the concept of 'roughness function' has been an active area of research in the engineering community (Perry et al., 1969; Flack and Schultz, 2014). In hydrology, the distinction between $z_{0 m}$ and $z_{0 s}$ over a variety of natural surfaces gained significant research attention with the proliferation of remote-sensing platforms that now will skin temperature at high spatial and temporal resolution (Schmugge et al., 2002; Morrison et al., 2020). Early analysis by Brutsaert (1975b) showed that scalar transfer differs for smooth and rough natural surfaces despite the fact that both are driven by molecular exchange. For a rough surface, it is now well established that $z_{0 m}$ and $z_{0 s}$ are not equal (Garratt, 1992, Chap.4). Similarity between turbulent transport of momentum and scalars (i.e. the Reynolds analogy) is generally valid for high Reynolds number ( $R e$ ) flows (Yang and Abkar, 2018), thus justifying the existence of a logarithmic layer for a passive scalar in Eq. 2. However, inequality between $z_{0 m}$ and $z_{0 s}$ still arises because in the 'immediate vicinity' of the wall (Brutsaert, 1975a), (i.e. the interfacial sublayer between the fluid and surface of the roughness elements), the mechanisms of scalar and momentum transfer differ. While scalar transfer relies on molecular diffusion, momentum transfer is dominated at high $R e$ by pressure (or form) drag in addition to the viscous drag (Brutsaert, 1975a,b).

Conventionally, $z_{0 s}$ is related to $z_{0 m}$ using the quantity $\kappa B^{-1}=\log \left(z_{0 m} / z_{0 s}\right)$ (Owen and Thomson, 1963; Garratt, 1992) because of the large variations expected in $z_{0 m} / z_{0 s}$. Although it is less discussed in the literature, $d$ and $d_{s}$ may also be unequal if momentum sinks and scalar sources/sinks drastically differ when eddy penetration into roughness elements is incomplete (Siqueira and Katul, 2010). Finally, roughness length concepts extend beyond the framework of MOST. Roughness lengths are indispensable model parameters when using the aerodynamic resistance approach 
(ARM), which is ubiquitously applied in various subfields of meteorology (Lhomme, 1991) and in common schemes to compute surface fluxes in mesoscale numerical weather models (Chen et al., 2010; Chen and Zhang, 2009).

\subsection{Roughness lengths for urban rough surfaces}

For context, previous work linking $z_{0 m}$ and $z_{0 s}$ to geometric features of urban rough surfaces is briefly reviewed. Emphasis is placed on $z_{0 s}$ because fewer studies on scalar roughness lengths over urban surfaces have been presented compared to momentum, despite their significance for turbulent fluxes in urban land-atmosphere models (Demuzere et al., 2008; Ryu et al., 2011; Wang et al., 2013; Li and Bou-Zeid, 2014). It is to be noted that roughness length models derived for vegetated canopies may not be directly extrapolated to urban surfaces. Buildings are bluff roughness elements that distinctly impact the flow when compared to permeable plants. Furthermore, the spatially heterogeneous surface temperatures and distributions of scalar sources/sinks within urban rough surfaces makes the problem of scalar roughness length determination a formidable challenge (Voogt and Grimmond, 2000; Kanda et al., 2007; Crawford et al., 2018). Nevertheless, this is a challenge that cannot be overlooked. It is of practical interest to determine $z_{0 m}, z_{0 s}$, and the corresponding bulk transfer coefficients over bluff-rough surfaces by relating them to directly-measurable surface geometrical properties (Kastner-Klein and Rotach, 2004; De Ridder, 2006; Kanda et al., 2007; Demuzere et al., 2008; Kanda et al., 2013; Zhu and Anderson, 2018). For $z_{0 m}$, significant progress has been achieved on this front (Macdonald et al., 1998; Grimmond and Oke, 1999; Barlow and Coceal, 2009; Kanda et al., 2013; Yang et al., 2016; Kent et al., 2017; Yang et al., 2019; Zhu and Anderson, 2018) and reviewing all this literature is beyond the scope of the work here. For $z_{0 s}$, the common approach is to relate it to $z_{0 m}$ using the quantity $K B^{-1}$ that is assumed to vary only with the roughness Reynolds number, $R e^{*}=u_{*} z_{0 m} / v$, where $v$ is the kinematic viscosity and $u_{*}$ is an effective friction velocity for the urban boundary layer. However, determining this relation $\kappa B^{-1}=f\left(R e^{*}\right)$ so as to obtain $z_{0 s}$ remains difficult.

Semi-idealized field experiments using an outdoor scale-model have allowed some progress to be made (Kanda et al., 2007). In terms of the geometric effects on scalar transport, it was shown (Kanda et al., 2007) that $K B^{-1}$ follows a universal parameterization based on a model by Brutsaert (1982) even for surfaces of different geometries. One limitation of such semi-idealized experiments is that they cannot capture other known factors that contribute to the variability of $K B^{-1}$ such as inhomogeneous distributions of heat sources and water vapor availability as reported in field experiments at a suburban site in Tokyo, Japan (Moriwaki and Kanda, 2006a). Furthermore, vegetation fraction and anthropogenic heat sources have been shown to impact roughness parameterization (Crawford et al., 2018) but these elements were not represented in scale-model experiments. Field experiments conducted at actual urban sites face daunting challenges as well. It has been demonstrated (Voogt and Grimmond, 2000) that uncertainties related to different methodologies of surface temperature determination influence the calculated value of $K B^{-1}$. In addition to field experiments, wind-tunnel experiments on bluff-bodies that are heated or coated with a passive scalar have also been used for roughness length studies. For instance, cuboids coated with naphthalene (Barlow et al., 2004) or wetted with water (Ikegaya et al., 2012; Chung et al., 2015) were used to investigate the dependence of scalar transfer coefficients on roughness packing density, height variations, orientations and distributions of scalar sources.

Numerical approaches such as large eddy simulations (LES) are increasingly used to tackle this problems as well. Anderson (2013) simulated flow and scalar transport over different fractal surfaces finding that the usually-assumed constant value for $K B^{-1}$ does not hold over the range of $R e_{*}=10-300$. This range encompasses aerodynamically rough morphologies often encountered in the atmospheric boundary layer, except for urban morphologies. Uncertainties are invariably associated with using numerical simulations over rough walls. In particular, wall-modeled LES, which is usually applied for simulating flows in the atmospheric boundary layer, does not resolve the viscous sublayer or the 
interfacial layer for each facet. Thus, molecular mass and heat diffusion that occurs at the interface between air and the surface of the roughness elements needs to be modeled. Wall-modeled LES is currently viewed as a leading numerical technique that is able to preserve the high $R e$ of the atmosphere. Unlike momentum transfer studies where the flow becomes Reynolds number independent beyond a certain high $R e$ threshold, matching the elevated $R e$ expected in the atmosphere has been shown to be necessary when exploring scalar transfer and its connection to momentum transfer (Li et al., 2016b).

Numerical experiments using LES over surfaces of different geometries are conducted here to address two research objectives: the first is to examine the implications of using wall-modeled LES to infer macro-scale scalar roughness length, and the second is to determine the scaling between $K B^{-1}$ and $R e^{*}$ and how it might depend on the geometric parameters of the rough surface. To address these two objectives, the problem (Section 2) is investigated by using LES results on roughness lengths for different surface geometries (Section 3). The implications on scalar roughness length parameterization using surface renewal theory (Section 4) are discussed prior to the summary and conclusions being drawn (Section 5).

\section{2 | METHOD}

\subsection{LES and roughness lengths}

LES is a common technique that is widely used to simulate turbulent flow and transport over urban terrain (Kanda et al., 2004; Bou-Zeid et al., 2009; Giometto et al., 2016). Direct numerical simulations (DNS), while preferred, cannot attain sufficiently high $R e_{b}=u_{*} \delta_{b l} / v$ values representative of the atmospheric boundary layer (Slotnick et al., 2014; Li et al., 2016b), where $\delta_{b l}$ is the boundary layer depth. The LES code employed here solves the equations of conservation of total mass, momentum (excluding the Coriolis force), and scalar mass or thermal energy. Buildings are represented using the immersed-boundary method (Appendix A). The scalar selected here is temperature. While temperature is not a passive scalar per se and buoyancy forces could arise, we simply omit the buoyancy force term from the vertical momentum equation to simulate a passive scalar in this study (i.e. the role of buoyancy remains to be explored). A constant temperature $(=330 \mathrm{~K})$ is applied to all solid surfaces, as an input to a wall-model for LES (see Eq. 4 ) as the scalar boundary condition. However, real buildings have neither constant temperature nor flux. Use of more realistic boundary conditions (e.g. coupling building energy models and radiative heat exchanges on the exterior surfaces) is left for future study.

Two different representations of roughness lengths must be clarified. As indicated in Fig. 1, within the urban boundary layer (hereafter macro-scale) roughness lengths $z_{0 m}^{m a c}$ and $z_{0 s}^{m a c}$, as well as the zero-plane displacements, are manifestations of the aggregate effects of the bluff-rough surfaces on the inertial sublayer aloft, where the logarithmic mean velocity and mean air temperature profiles hold. (e.g. (Kanda et al., 2013; Yang et al., 2016)) macro-scale thermal roughness lengths for flows over steep ocean waves (Sullivan et al., 2017). This needs to be distinguished from each single building facet's (e.g. a roof) boundary-layer (hereafter micro-scale) roughness parameters. The micro-scale roughness is associated with its own local velocity and temperature distribution near the surface, which follow a micro-scale log-law but only in regions where the flow is facet-parallel and in equilibrium with the adjacent surface. These micro-scale roughness and boundary layers in high- $R e$ wall-modeled LES cannot be resolved (Pope, 2000), and require parameterization supplied as a wall model formulation (Li et al., 2016b). Examples of such micro roughness include subgrid-scale wind ripples on resolved waves (Sullivan et al., 2017) and small indentations on the surfaces of built structures (Cai, 2012; Li et al., 2016b), to name a few. To add to the difficulty of the problem, it is well established that the surface heat exchange parameterized using a wall model, and consequently the flow over an urban canopy, are 
sensitive to specification of the micro-scale thermal roughness length (Cai, 2012). Therefore, if one aims to determine the macro-scale roughness length, an evaluation of the parameterization of the micro-scale exchanges is required.

To simplify this problem within the confines of LES, the micro-scale roughness elements are assumed to be smaller than the depth of the local viscous sublayer attached to building facets such that the LES work focuses on the macroscale. This simplification is akin to setting all building facets as smooth walls. For an aerodynamically smooth surface in the absence of buoyancy forces, the wall-models for momentum and scalar to be used in the LES can be expressed as

$$
u_{*}^{w m}=\frac{\kappa u}{\log \frac{z}{z_{0 m}^{m i c}}},
$$

$$
\theta_{*}^{w m}=\frac{\kappa \Delta \theta}{\log \frac{z}{z_{0 s}^{m i c}}},
$$

where the superscript $w m$ represents the wall-modeled quantities of surface stress and scalar flux $F_{s}=u_{*}^{w m} \theta_{*}^{w m} ; \Delta \theta$ is the difference between $\theta$ at the surface and at the first grid point away from the wall (where the flow is assumed to be already fully turbulent); and $u$ is the wall-parallel fluid velocity at that same first grid point. The micro-scale roughness lengths $z_{0 m}^{m i c}$ and $z_{0 s}^{m i c}$ are set as

$$
z_{0 m}^{m i c}=\frac{1}{9} \frac{v}{u_{*}^{w m}}, \quad z_{0 s}^{m i c}=\frac{1}{S c} z_{0 m}^{m i c}
$$

where the constant 9 is obtained from experiments (Kader and Yaglom, 1972), $S c=v / D_{m}$ is the molecular Schmidt number set to unity for simplicity here and $D_{m}$ is the molecular diffusion coefficient for the scalar of interest. Hence, at the micro-scale, the LES assumes $z_{0 m}^{m i c}=z_{0 s}^{m i c}$. To be clear, such an LES parameterization does not resolve the viscous sublayer and does not capture the turbulence disequilibrium as the mean flow meanders around the buildings (at the micro-scale), and as such it cannot be an exact model. Log-law wall models are only accurate if the grid point nearest to the facet is within its equilibrium layer; this should hold over most of the facet areas but might be less accurate near transitions and edges. However, previous evaluation of this model for roughness elements with smooth facets indicate that it is adequate for the present purposes (Li et al., 2016b). The aim here is not to refine such wall models in the LES, which continues to be a formidable challenge (Bose and Park, 2018). Rather, the goal is to examine the implications of the aforementioned wall-model on the macro-scale scalar roughness problem with a lens on $k B^{-1}$.

\section{2 | Simulation cases}

The details about the LES code are provided in Appendix A and references therein. The grid resolution of the current setup is able to resolve the geometry of the obstacles as for most runs the number of grids resolving one obstacle is greater than six points, as shown to be sufficient with a similar code (Tseng et al., 2006). Sensitivity analysis performed for case VF32 (Li and Bou-Zeid, 2019) found all quantities relevant for computing the scalar and momentum displacement heights and roughness lengths change by less than $6 \%$ when the resolution is doubled. Thus, we use the current setup to allow the parameter space to be finely addressed with a reasonable computational cost.

Three categories of urban forms with different plan area density $\left(=\lambda_{p}\right)$ and frontal area density $\left(=\lambda_{f}\right)$ are considered. 
(a)

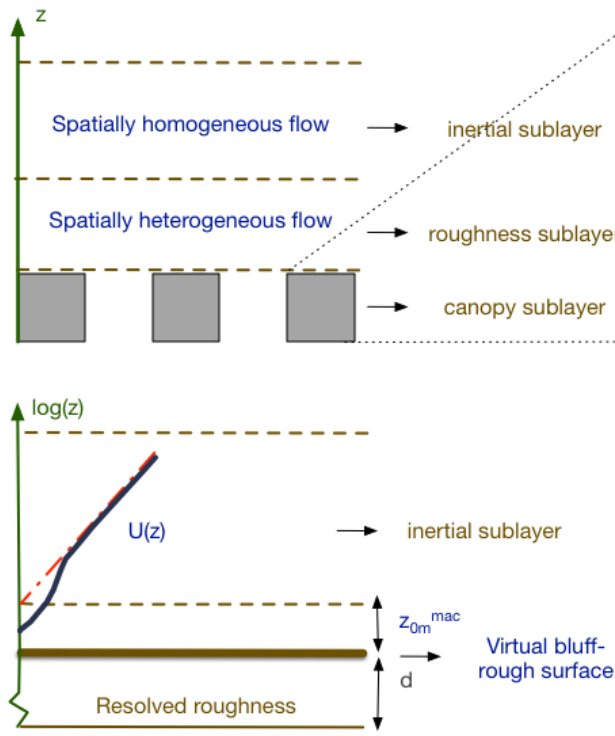

(b)

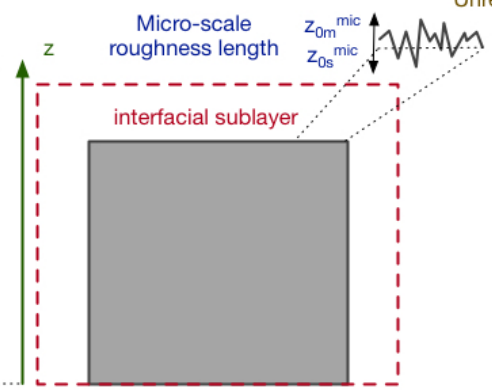

(d)

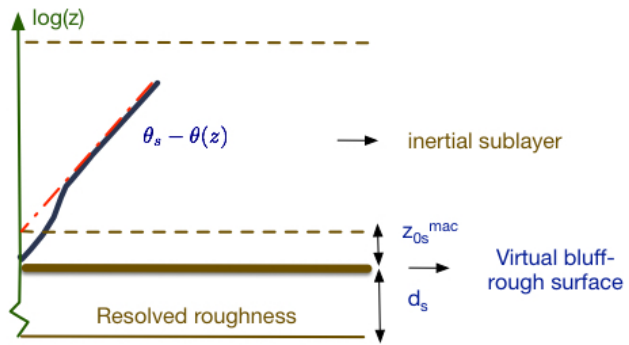

FIGURE 1 Schematic illustration of micro-scale and macro-scale roughness lengths in the LES. The LES resolves the bluff bodies present in the canopy sublayer in (a) but the facet-specific roughness elements shown in (b) are unresolved and must be represented using a wall-model of the interfacial sublayer. The micro-scale roughness lengths are imposed or parameterized in the wall model. The macro-scale roughness lengths for momentum (c) and scalar (d) have virtual origins of the logarithmic profiles of average $\langle u\rangle$ and $\left\langle\theta_{s}-\theta\right\rangle$. The $d$ and $d_{s}$ define the "virtual bluff-rough" surfaces that represent the aggregate effects of the resolved roughness elements. Notation is defined in the text. 
First, (figure 2a), $\lambda_{p}$ and $\lambda_{f}$ are varied simultaneously but with $\lambda_{p}=\lambda_{f}$ hereafter "Variable Plan and Frontal area density" (VPF). Second (figure $2 \mathrm{~b}$ ), $\lambda_{f}$ is maintained constant at 0.25 but $\lambda_{p}$ is varied (Variable Plan area density, VP). Third (figure 2c), $\lambda_{p}$ is kept constant at 0.12 but $\lambda_{f}$ is varied (Variable Frontal area density, VF). An empirical quadratic relation between $\lambda_{f}$ and $\lambda_{p}$ derived from Japanese building data (Kanda et al., 2013) found appreciable scatter particularly for non-Japanese cities for fixed $\lambda_{p}$ (see their Figure 2). Hence, the category VF explores the effect of such scattered $\lambda_{f}$ at a preset fixed $\lambda_{p}$. The simulation parameters for the various cases are summarized in Table 1 . The focus is on the parameter space where the two characteristic densities fall between 0.1 and 0.5 , reflecting the expected values for many cities and districts (Kanda et al., 2013).

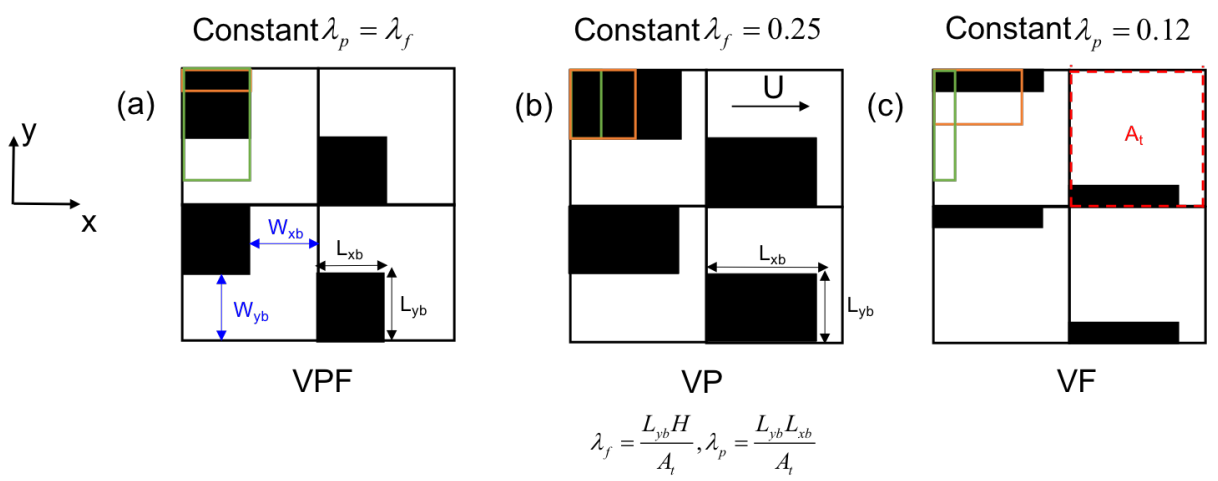

FIGURE 2 Plan area view of one "repeating unit" consisting of four buildings (black) as different geometries with a height $(H)$ of $12.5 \mathrm{~m}$. The black, orange and green lines illustrate three different possible variations of geometry within each category. (a) Variable plan and frontal area VPF; (b) Variable plan at a constant frontal area VP; (c) Variable frontal area at a constant plan area VF. The dimensions of the repeating units, $D_{x} \times D_{y}$, are $0.5 \delta \times 0.5 \delta$, $0.5 \delta \times 0.5 \delta$ and $0.625 \delta \times 0.625 \delta$ in VPF, VP and VF, respectively (see Table 1). $L_{x b}$ and $L_{y b}$ are the dimensions of the obstacles in the $x$ and $y$ directions; $W_{x b}$ and $W_{y b}$ are distances between adjacent obstacles in the $x$ and $y$ directions. Notice that for all cases $W_{x b}+L_{x b}=W_{y b}+L_{y b}=D_{x}=D_{y}$.

\section{3 | ROUGHNESS LENGTHS DETERMINED FROM LES}

Selected examples of the profiles of horizontally-averaged $\langle\bar{u}\rangle / u_{*}$ and $\left\langle\left(\overline{\theta_{s}}-\bar{\theta}\right)\right\rangle / \theta_{*}$ are illustrated in Fig. 3 for the VPF, VP and VF categories. Although a least-square fitting procedure to the log-law is a common approach to determine roughness lengths and displacement height (Kanda et al., 2004; Bou-Zeid et al., 2009; Kanda et al., 2013; Placidi and Ganapathisubramani, 2015; Yang et al., 2016). we use a 'brute-force' global parameter search in the $d / H$ $z_{0 m} / H$ parameter space. Additionally, $z_{0 m} / H<d / H$ can be enforced when searching all parameter combinations. The optimal $z_{0 m} / H$ and $d / H$ parameters that best fit the pre-normalized velocity $\langle\bar{u}\rangle / u_{*}$ (angled brackets indicate spatial averaging) are those that yield a minimum root-mean-squared error (RMSE) over the range $1.25 \leq z / H \leq 2.25$. The results and conclusions are robust to the choice of logarithmic region to perform the fitting procedure. A sensitivity analysis to the fitting range in the log-layer is shown in Appendix C; maximum deviations at the extreme fitting ranges are less than $40 \%$ relative to the adopted ranges. For all these calculations, $k=0.4$. For notational simplicity, superscripts of macro-scale roughness length are omitted. Different procedures can lead to different computed values for $d$ (Leonardi and Castro, 2010) and consequently of $z_{0 m}$. For example, the "drag force moment" approach to calculate 


\begin{tabular}{|ccccccc} 
Case & $\lambda_{p}$ & $\lambda_{f}$ & $\sum_{i} \lambda_{i}$ & $N_{x}^{b}, N_{y}^{b}, N_{z}^{b}$ & $N_{x}, N_{y}, N_{z}$ & $R e_{*}$ \\
\hline VPF12 & 0.125 & 0.125 & 1.75 & $8,4,8$ & $192,96,64$ & 21250 \\
\hline VPF18 & 0.1875 & 0.1875 & 1.87 & $8,6,8$ & $192,96,64$ & 42930 \\
\hline VPF25 & 0.25 & 0.25 & 2 & $8,8,8$ & $192,96,64$ & 49160 \\
\hline VPF31 & 0.3125 & 0.3125 & 2.12 & $8,10,8$ & $192,96,64$ & 36290 \\
\hline VPF37 & 0.3725 & 0.3725 & 2.25 & $8,12,8$ & $192,96,64$ & 34910 \\
\hline VPF43 & 0.4375 & 0.4375 & 2.375 & $8,14,8$ & $192,96,64$ & 14096 \\
\hline VP12 & 0.125 & 0.25 & 1.75 & $4,8,8$ & $192,96,64$ & 88750 \\
\hline VP18 & 0.1875 & 0.25 & 1.875 & $6,8,8$ & $192,96,64$ & 70860 \\
\hline VP25 & 0.25 & 0.25 & 2 & $8,8,8$ & $192,96,64$ & 49160 \\
\hline VP31 & 0.3125 & 0.25 & 2.125 & $10,8,8$ & $192,96,64$ & 48220 \\
\hline VP43 & 0.4375 & 0.25 & 2.375 & $14,8,8$ & $192,96,64$ & 17850 \\
\hline VF08 & 0.12 & 0.08 & 1.76 & $12,4,8$ & $200,100,64$ & 9770 \\
\hline VF12 & 0.12 & 0.12 & 1.64 & $8,6,8$ & $200,100,64$ & 35850 \\
\hline VF16 & 0.12 & 0.16 & 1.56 & $6,8,8$ & $200,100,64$ & 78740 \\
\hline VF24 & 0.12 & 0.24 & 1.56 & $4,12,8$ & $200,100,64$ & 16504 \\
\hline VF32 & 0.12 & 0.32 & 1.64 & $3,16,8$ & $200,100,64$ & 16009 \\
\hline
\end{tabular}

TABLE 1 Summary of simulation parameters: $N_{i}^{b}$ is the number of nodes resolving one obstacle and $N_{i}$ is the total number of nodes for the entire domain in the $i$ direction ( $i=1$ is $x$ or longitudinal direction, $i=2$ is $y$ or lateral direction and $i=3$ is $z$ or vertical direction). The number of nodes resolving the repeating units of dimensions $D_{x} \times D_{y}$ are $32 \times 32,32 \times 32$ and $40 \times 40$ for VPF, VP and VF, respectively, where $V$ is 'variable', $P$ is 'plan' and $F$ is 'frontal' area; and the last two digits represent the first two decimal values of $\lambda_{p}$ or $\lambda_{f}$. $R e_{*}=u_{*} z_{0 m} / \nu$, where $u_{*}$ is $\sqrt{-\left\langle\overline{u^{\prime} w^{\prime}}\right\rangle}$ defined at $z / H=1.5$ and $v$ is kinematic viscosity. Dimension of the computational domain normalized by the boundary layer height $\delta$ is $L_{x} / \delta=3.0, L_{y} / \delta=1.5, L_{z} / \delta=1$ in cases VPF and VP; $L_{x} / \delta$ $=3.125, L_{y} / \delta=1.5625, L_{z} / \delta=1$ in cases VF. 

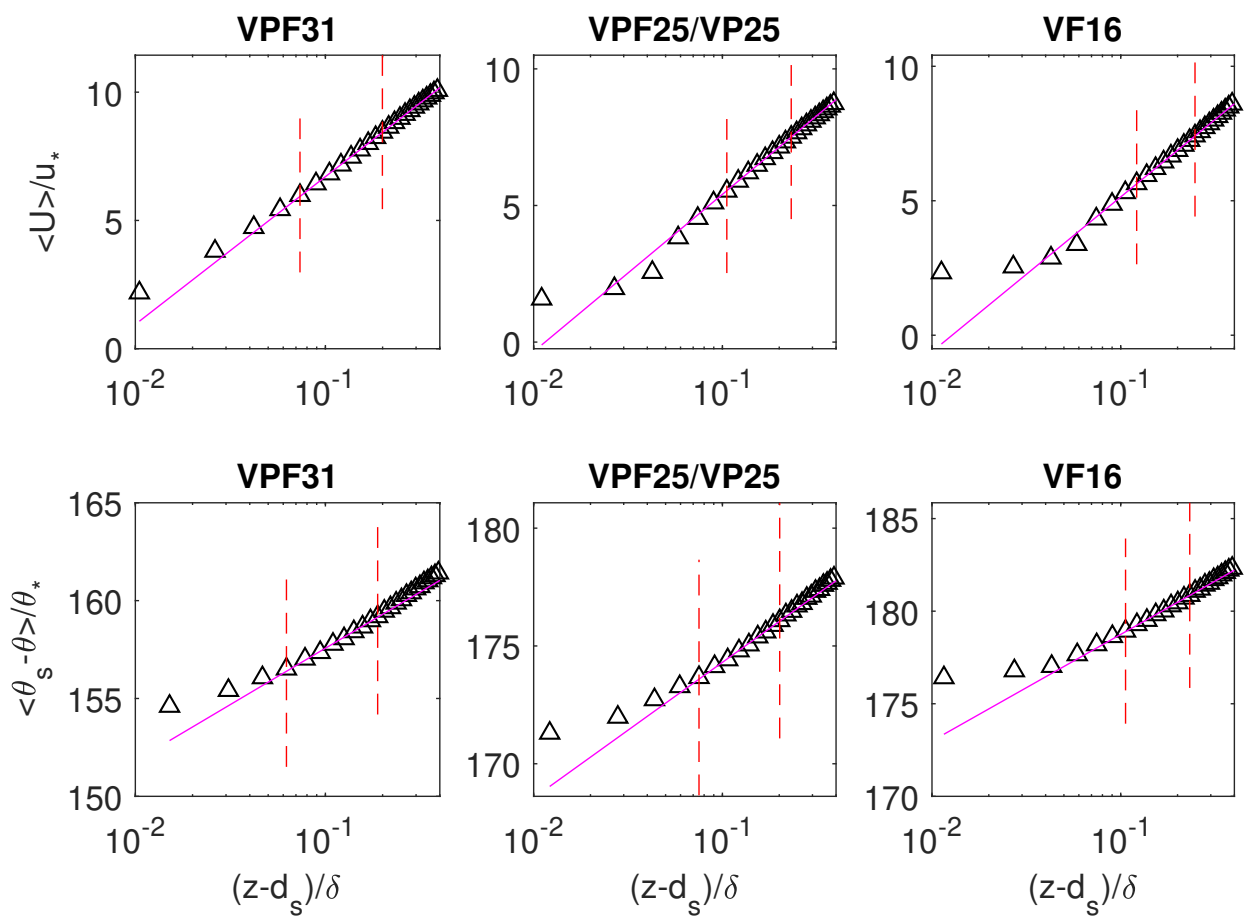

FIGURE $3\langle\bar{u}\rangle / u_{*}$ and $\left\langle\left(\overline{\theta_{s}}-\bar{\theta}\right)\right\rangle / \theta_{*}$ for three selected cases from the VPF, VP and VF, categories $(V$ variable, $P$ plan and $F$ for frontal area). The log-law region from $z / H=1.25$ to 2.25 (red dotted vertical lines) indicate the log-lawsobtained from the fitting procedure (magenta solid lines). 
$d$ (Jackson, 1981) and other approaches are discussed (Leonardi and Castro, 2010). As the methodology selected only have minor impact on the the momentum and scalar roughness lengths (Appendix $\mathrm{C}$ ), it is not explored further in this study. Given the minor differences between $d$ and $d_{s}$ and minor influence on the deduced scalar roughness length, the $z_{0 s}$ is determined with $d_{s}=d$ (reducing the degrees of freedom in roughness length parameterizations). The densest case VP case has a $d / H$ around unity (or slightly larger). This can be attributed to pressure drag effects extending above $H$, or to uncertainty in the fitting procedure. Using a von Kármán constant $(\kappa)$ of 0.4 , Kanda et al. (2013) found a higher $d$ than adopting a variable $\kappa$.

For comparison, two models of $d$ and $z_{0 m}$ (Macdonald et al., 1998; Kanda et al., 2013) are featured in Fig. 4. At low $\lambda_{f}$, there are significant differences in predicting $d$ (both models assume $d$ does not vary with $\lambda_{f}$ although the LES results suggest it does), particularly for the Macdonald et al. (1998) model (Fig. 4f). The two models predict a decline in $z_{0 m}$ with increasing $\lambda_{f}$ and decreasing $\lambda_{p}$, consistent with the LES-inferred values. The two-parameter $\left(\lambda_{p}\right.$ and $\left.\lambda_{f}\right)$ Macdonald et al. (1998) model surprisingly captures the responses of $z_{0 m}$ to such variable geometries considered in the LES runs (even though it failed to predict $d$ ). The surface geometries in our study fall in Mcdonald et al.'s (1998) 'dense regime' (i.e. $\lambda_{f}>0.03$ ) for all cases. The Kanda et al. (2013) model performs better overall when both $d$ and $z_{0 m}$ are considered. For realistic highly-heterogeneous urban morphologies, more sophisticated modeling may be required. In such complex canopies, the models might need to explicitly account for the mutual interaction between roughness elements (Yang et al., 2016), or for additional statistical properties of the rough surface such as building height variance or skewness (Kanda et al., 2013; Zhu et al., 2017). Nevertheless, a morphometrically-based model appears viable to estimate $z_{0 m}$.
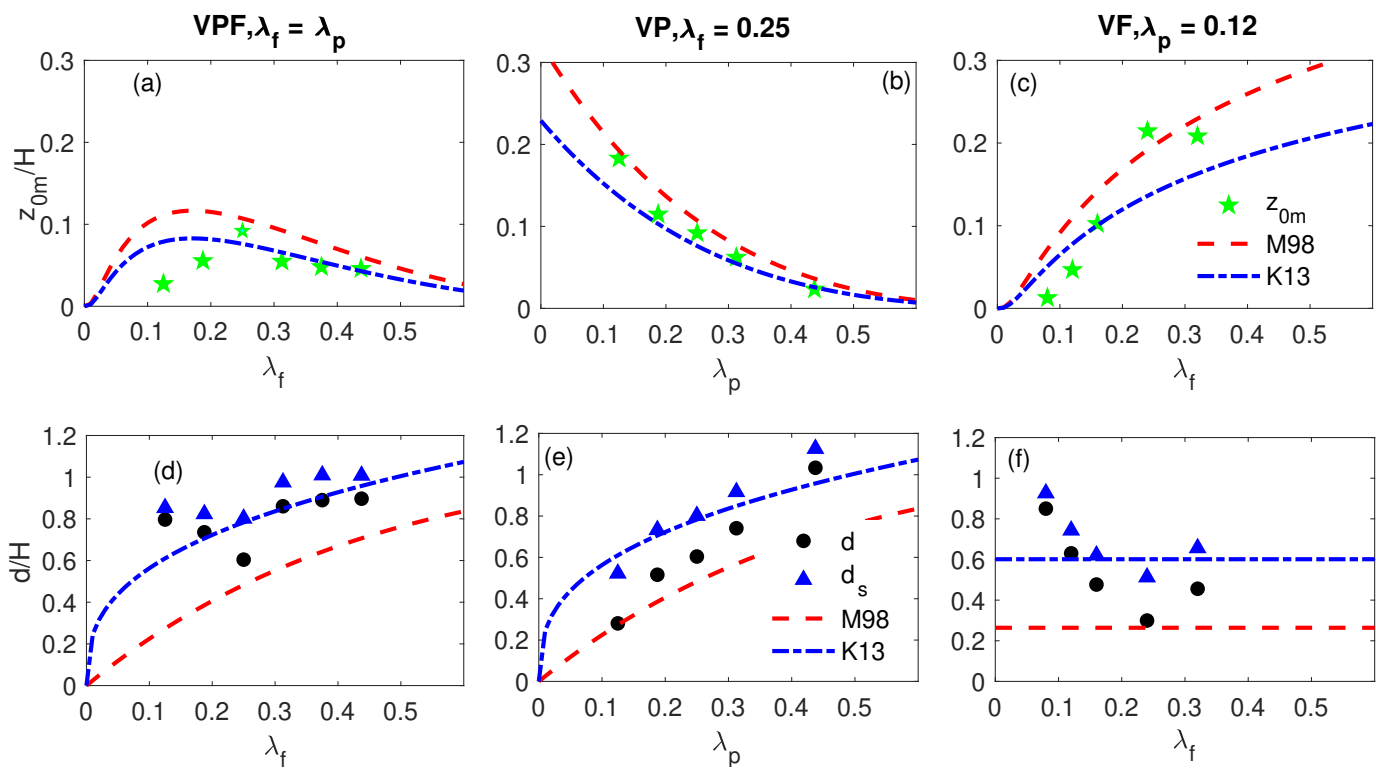

FIGURE 4 Variation of $z_{0 m}$ and $d$ determined by direct fitting to LES output for $z / H=1.25-2.25$. (a)-(c): $z_{0 m}$ and (d)-(f): $d$. Macdonald et al. (1998) (red-dotted line) and Kanda et al. (2013) (blue-dash-dotted line) models are also shown for categories: (a,d) VPF; (b,e) VP; (c,f) VF. 
to explain the non-monotonic behavior in the VPF cases $\left(\lambda_{p}=\lambda_{f}\right)$. Because of the opposing effects, a peak in $z_{0 m}$ is expected and occurs between $\lambda_{p}=\lambda_{f}=0.25$ and 0.31 (see Fig. 4a). This peak in $z_{0 m}$ has been observed in many previous studies (Kanda et al., 2004; Hagishima et al., 2009; Leonardi and Castro, 2010) for arrays of cubical roughness in which $\lambda_{p}$ and $\lambda_{f}$ are set equal. Such non-monotonic behavior was also noted by Macdonald et al. (1998) as the obstacles become more densely packed. The peak value of $z_{0 m} / H$ is around 0.1 (Fig. 4), which is different from the cubical arrays considered by previous studies (Leonardi and Castro, 2010) reporting the peak value of $z_{0 \mathrm{~m}} / H$ to be around 0.15 . This is likely caused by the obstacles in VPF not being cubes with equal dimensions in all directions, except for VPF25.

The variations of $z_{0 s}$ (Fig. 5a-c) between different cases overwhelmingly outweigh the effects of changes in $z_{0 s}$ when $d$ or $d_{s}$ are used in the fitting. Values of $z_{0 s}$ computed using $d$ differ from those using $d_{s}$ by less than $10 \%$ for more than half of the cases (with a highest deviation of 20\%). Such differences do not change the trend of variation across cases and produce negligible change in the values of $\log \left(z_{0 m} / z_{0 s}\right)$ (at most $0.3 \%$ ). Our assumption $d=d_{s}$ is justifiable for the boundary conditions imposed here. As with $z_{0 m}, \lambda_{p}$ and $\lambda_{f}$ have inverse impacts on $z_{0 s}$, but are exactly opposite to that noted for $z_{0 m}$. The $z_{0 s}$ increases with increasing $\lambda_{p}$ and decreasing $\lambda_{f}$.
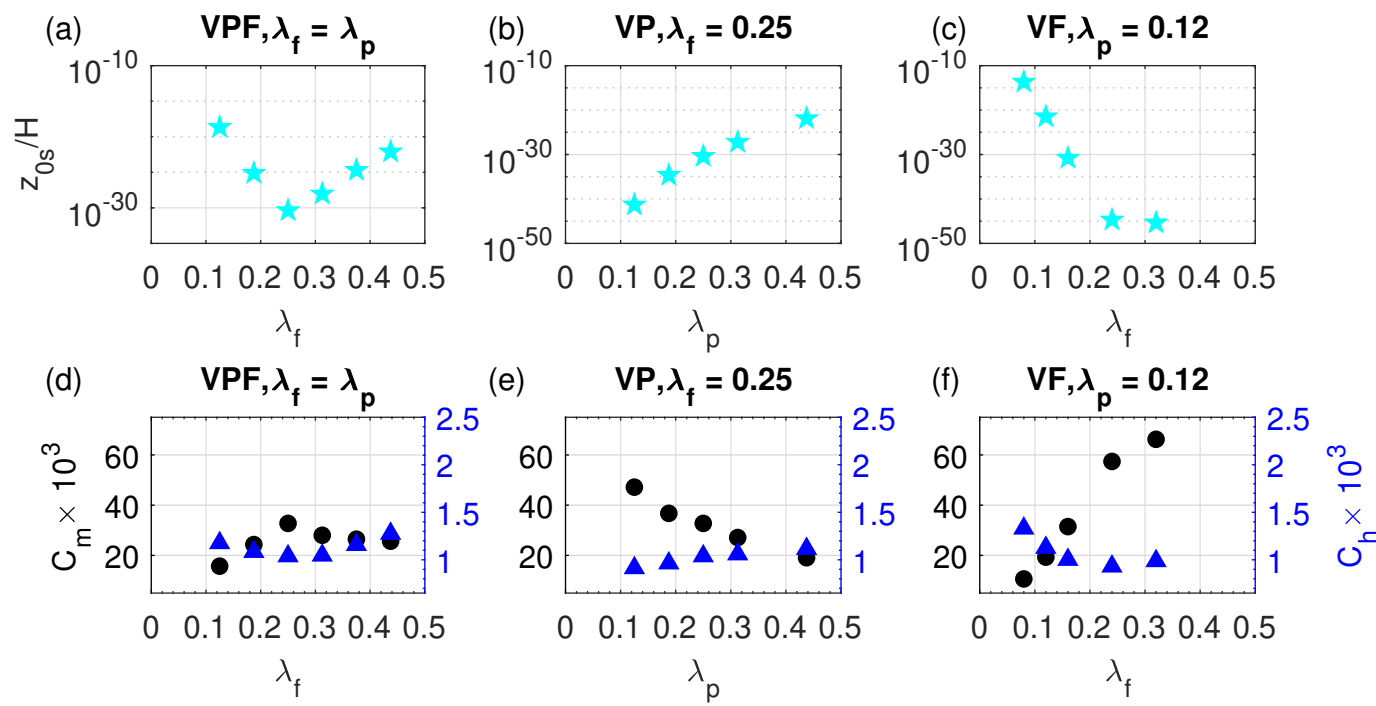

$\begin{array}{llllll}0 & 0.1 & 0.2 & 0.3 & 0.4 & 0.5\end{array}$

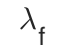

FIGURE 5 (a)-(c): The $z_{0 s}$ determined from least-square error minimization using $d$. (d)-(f): The bulk transfer coefficients for momentum $\left(C_{m}\right)$ and scalar $\left(C_{h}\right)$ computed at $z / H=1.5$.

The bulk transfer coefficients for momentum and scalar are defined as

$$
C_{m}(z)=\kappa^{2}\left(\log \left(\frac{z-d}{z_{0 m}}\right)\right)^{-2}
$$

and

$$
C_{h}(z)=\kappa^{2}\left(\log \left(\frac{z-d}{z_{0 m}}\right)\right)^{-1}\left(\log \left(\frac{z-d_{s}}{z_{0 s}}\right)\right)^{-1}
$$


and are shown in Figs. $5 \mathrm{~d}$-f for $z / H=1.5$. The $C_{m}$ and $C_{h}$ values are height dependent quantities (sometimes a factor of 2 is used in these definitions). The $C_{h}$ values obtained here are within the range of scalar transfer coefficients obtained from previous wind-tunnel experiments (Barlow et al., 2004; Chung et al., 2015). A scalar transfer coefficient was derived using two-dimensional street canyon and a naphthalene sublimation technique (Barlow et al., 2004) ranges from $1 \times 10^{-3}$ to $3.5 \times 10^{-3}$ (see their figure 4). Likewise, a $C_{h}$ that ranges between $3 \times 10^{-3}$ to $3.75 \times 10^{-3}$ was obtained from measurements of wetted surfaces using three-dimensional arrays of cubes (Chung et al., 2015). While uncertainty in the adopted scalar flux wall-model does affect $\theta_{*}$ and hence $C_{h}$, the determined $C_{h}$ seems to be robust with such uncertainty. The uncertainty from the wall modeling approach to $C_{h}$ is estimated to be approximately $25 \%$ (Li et al., 2016b). The differences might also be related to the lower $R e$ in the wind tunnel experiments. As the relative change in $C_{h}$ across the cases considered here exceeds $25 \%$, the morphological dependence, at least qualitatively, still holds. The $C_{m}$ and $C_{h}$ combine the effects of the displacement height and roughness length on the surface exchange effectiveness, and they show independent monotonic variation with $\lambda_{p}$ or $\lambda_{f}$. Their anti-correlated trends with respect to varying geometric parameters confirm that there can be significant "trade-off effects" in the effectiveness of a surface to exchange momentum or scalars with a turbulent flow.

From Eq. 7 the magnitude of $z_{0 s}$ can be understood, as $C_{h}$ depends on both $z_{0 s}$ and $z_{0 m}$ even with a constant $C_{h}$, if the wall gets rougher and both pressure drag and $z_{0 m}$ increase, $z_{0 s}$ must decrease. Therefore, $z_{0 s}$ alone is insufficient to understand wall heat transfer effectiveness ( $C_{h}$ is better for this task). Moreover, the very small magnitudes of $z_{0 s}$ do not imply a vanishingly small heat transfer, and $z_{0 s}$ should not be interpreted as a macroscopic physical length scale or morphometric parameter. One can note, for example, that the reported values here (and elsewhere) are smaller than the mean free path of atmospheric molecules (i.e. about $70 \eta \mathrm{m}$ ). This clarifies why determining $z_{o s}$ from morphological parameters remains a daunting task for urban canopies and other surfaces as well (at least when compared to $z_{o m}$ ).

As indicated, $z_{0 s}$ is commonly obtained from $z_{0 s} / z_{0 m}$ or $\kappa B^{-1}=\log \left(z_{0 m} / z_{0 s}\right)$ as a function of $R e_{*}$. Justified on the grounds that $z_{0 m}$ is reasonably described from simplified two-parameter models (e.g. $\lambda_{f}, \lambda_{p}$ ), as confirmed here. For a variety of surfaces, including bluff-rough surfaces, a relation of the form $z_{0 s} / z_{0 m} \sim R e_{*}^{n}$ is used (Brutsaert, 1965, 1975b; Zilitinkevich et al., 2001; Li et al., 2017; Katul and Liu, 2017; Castellví, 2018). A variety of exponents ( $n=0$, $1 / 4$ or $1 / 2$ ) and intercepts have been reported across differing surfaces. Their universal character is questioned in several studies. The next section discusses such parameterization using a phenomenological model based on surface renewal theory, which has rarely been applied to urban canopies. Its development here constitutes one of the main theoretical novelties of this paper.

\section{I SCALAR ROUGHNESS LENGTH FROM SURFACE RENEWAL THEORY}

Since Danckwerts (1951) first introduced a statistical model that accounts for duration over which eddies are in contact with an interface (i.e. interval between renewals), extending the surface renewal theory (SRT) to model turbulent transport of scalars in the atmospheric boundary layer has been the subject of inquiry for the past 40 years (Brutsaert, 1975b; Clayson et al., 1996; Katul et al., 1996; Snyder et al., 1996; Denby and Snellen, 2002; Katul et al., 2006; Castellvi et al., 2008; Li et al., 2017). Most applications of the SRT are limited to the "natural" rough and smooth surfaces such as the ocean surface (Clayson et al., 1996), glacier surfaces (Denby and Snellen, 2002), grasslands (Li et al., 2017) and forests (Katul et al., 1996). Whether the surface renewal concept is applicable to very rough surfaces that consist of large bluff-body objects such as buildings is now considered within the context of interfacial scalar transfer. 


\section{1 | Basics of Surface Renewal Theory}

The basic assumptions common to all SRT schemes are briefly reviewed. A parcel of air sweeps to the surface and is in contact with the surface for a random duration $s$. During the interval $s$, the parcel exchanges mass or heat with the surface via molecular diffusion only. After $s$, the parcel is ejected and is replaced by a new parcel. In the simplest SRT, this repeated sweep-ejection-replacement process leads to what is labeled as 'continuous surface renewal'. The distributional properties of $s, \phi(s)$ must be externally supplied and are assumed to follow an exponential form (Danckwerts, 1951). Other forms (e.g. log-normal, power-law, Gamma, Inverse-Gaussian) have also been proposed with various justifications (Katul and Liu, 2017). The choice $\phi(s)=R \exp (-R s)$ is the simplest and most convenient - it defines the probability density function for the rate of occurrence of a surface renewal event and satisfies the normalizing property $\int_{0}^{\infty} \phi(s) d s=1$. This one-parameter probability density function requires only the specification of $1 / R$. Physically, $1 / R=\int_{0}^{\infty} s \phi(s) d s$ is the mean time that an eddy remains in contact with the surface before it is ejected away. In general, $\phi(s)$ must only depend on the flow properties, not the scalar being analyzed. The exact functional form of $\phi(s)$ appears not to be as crucial as the specification of $1 / R$ - at least for mean flux calculations as discussed elsewhere (Katul and Liu, 2017). In all SRT versions, the scalar transfer at the surface over a contact duration $s$ is, as earlier noted, assumed to be via molecular transfer characterized by $D_{m}$ and a local gradient at the surface during event $s$. To a leading order, this near-surface concentration gradient during $s$ can be approximated by $\left(\Delta \theta^{\prime}\right) / \sqrt{D_{m} s}$, where $\Delta \theta^{\prime}$ is assumed to be primarily dominated by mean scalar changes within the local interfacial layer with a characteristic thickness $\sqrt{D_{m} s}$ (the layer bounded by red dotted line in Fig. 1b; i.e. at the micro-scale and accommodated only in the wall model of the LES).

With the choice of the distribution function $\phi(s)=R \exp (-R s)$, the mean thickness of the local interfacial layer is $L_{i}$ given by

$$
L_{i} \propto \int_{0}^{\infty} \phi(s)\left(D_{m} s\right)^{1 / 2} d s=\frac{1}{2} \sqrt{\frac{\pi D_{m}}{R}} .
$$

Mean temperature changes from $L_{i}$ to any height above $L_{i}$ are assumed to be minor when compared to $\Delta \theta^{\prime}$ within $L_{i}$. This is required in SRT because differences in temperatures of air parcels originating from outside this interfacial layer are not explicitly considered, and $\Delta \theta^{\prime}$ is thus strictly controlled by the surface flux. With this background, the heat flux summed over all random contact times must be given as

$$
F_{c}=-\int_{0}^{\infty} \phi(s) D_{m}\left(\frac{\partial \theta}{\partial z}\right)_{z=0} d s \propto-D_{m} \int_{0}^{\infty} R \exp (-R s) \frac{\Delta \theta^{\prime}}{\left(D_{m} s\right)^{1 / 2}} d s
$$

This expression can be arranged to yield

$$
S h=\frac{F_{c}}{u_{*} \Delta \theta^{\prime}}=\frac{1}{u_{*}} A \pi D_{m}{ }^{1 / 2} R^{1 / 2}
$$

where $S h$ is the Sherwood number (a type of a mass transfer Stanton number) and $A$ is a proportionality constant. When the total scalar or heat fluxes and $u_{*}$ are constant with $z$ and the primary $\Delta \theta^{\prime}$ is across the interface, the $S h$ can be interpreted as both an interfacial and a bulk (i.e. extending to the inertial layer, not just the interfacial layer) characteristic. These assumptions are common to virtually all SRT. The main difference between various SRT approaches is broadly in how $1 / R$ is specified. It is thus instructive to analyse these approaches using two 'endmember' cases: the micro-eddy and macro-eddy models (Fig. 6) characterizing $1 / R$. 


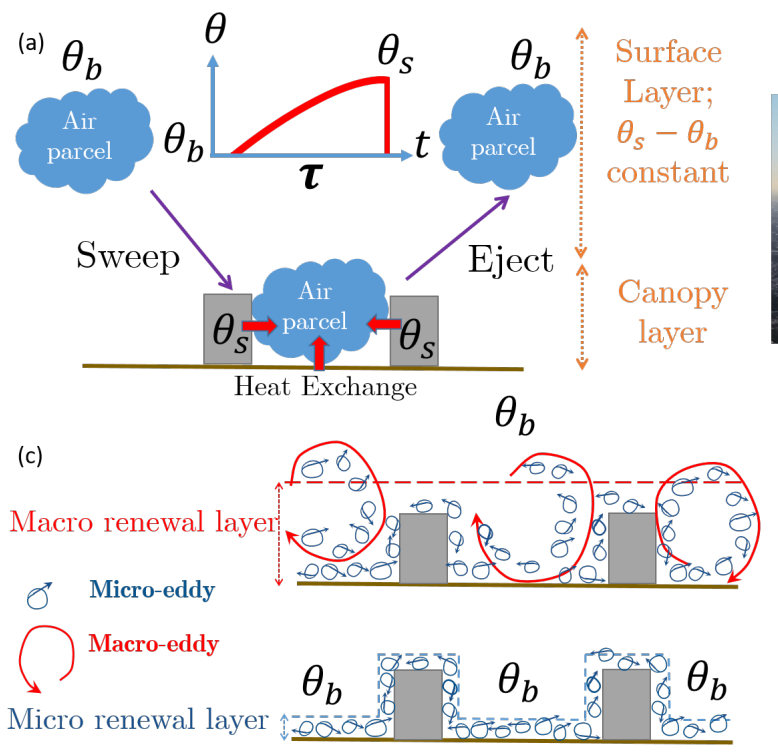

(b)

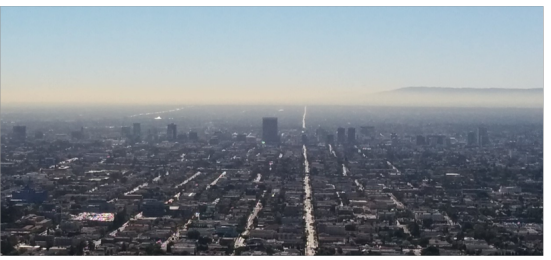

(d)

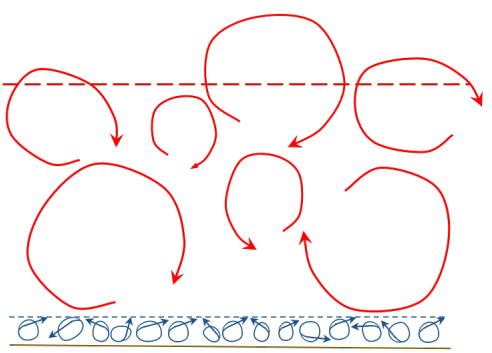

FIGURE 6 A schematic illustrating the (a) continuous surface renewal theory (SRT) process and (b) a typical urban bluff-rough surface. (c) concepts of the micro-scale and macro-scale eddies viewed in the context of SRT, and (c) with bluff bodies and (d) without bluff bodies. For the micro-eddy model, it is assumed that the limiting step in the scalar exchange process is availability of Kolmogorov eddies that can populate the entire surface area. For the macro-eddy model, the ability to produce or bring Kolmogorov eddies to the exchanging surface by macro-eddies is considered to be the limiting step. 


\subsection{The micro-eddy model}

In the micro-eddy model, it is assumed that large eddies can be efficient in bringing air parcels (and small eddies) near the solid interface but do not interact with the interface. Heat or mass exchange at the solid interface must only occur after air parcels penetrate and momentarily break-up the viscous sublayer and make direct contact with the solid surface. Hence, we take the view that only the smallest of eddies (active in the viscous and buffer layers) are sufficiently efficient at penetrating the viscous sublayer to interact with the surface. According to the micro-eddy model, this efficiency is not based on the energy content of an eddy. If an eddy is sufficiently energetic to penetrate into or displace the viscous sublayer, any excess kinetic energy carried by the eddy is not used in the renewal and its interaction with the surface will not depend on this excess. Hence, the micro-eddy model assumes that it is the collision frequency between eddies and the viscous sublayer that dictates the renewal frequency, not the energy content of eddies. Very small eddies in the vicinity of the interface are expected to experience collision events with the viscous sublayer at a high frequency when compared to large eddies. Because of the high frequency of collisions, the very small eddies encounter the viscous sublayer far more frequently (at least compared to their much larger eddies counterparts) and contribute to scalar mass exchange. In the energy cascade, these small eddies are commensurate in size with the so-called Kolmogorov viscous length scale (Brutsaert, 1965). Under such a condition, $1 / R$ is assumed to scale with the Kolmogorov time scale $=(v / \epsilon)^{1 / 2}$, where $\epsilon$ is the mean turbulent kinetic energy dissipation near the interface. With $R=(v / \epsilon)^{-1 / 2}$ (flow and fluid properties) and $D_{m}=S c^{-1} v$,

$$
S h=\frac{F_{c}}{u_{*} \Delta \theta^{\prime}}=\frac{1}{u_{*}} A\left(\pi S c^{-1}\right)^{1 / 2}(v \epsilon)^{1 / 4}
$$

where $(v \epsilon)^{1 / 4}$ is the Kolmogorov velocity with an associated kinetic energy $=(v \epsilon)^{1 / 2}$.

This micro-eddy model thus postulates that the canyon sublayer air in between the buildings has comparable scalar concentration to the roughness and inertial layer air, and that the micro eddies then sweep this canopy layer air in contact with various facets where it is further enriched in scalar concentrations. To place this result in the context of prior theories (Brutsaert, 1965), the following representation of the cuboid surface can also be made. The cuboids-air interface is first replaced by an equivalent rough surface (i.e. experiencing the same scalar flux and $u_{*}$ ) characterized by $z_{0 m}$ and positioned at $z=d$ (Fig. 1c thick brown line). For this rough surface, the turbulent kinetic energy dissipation rate inside the equivalent roughness elements (characterized by $z_{0 m}$ ) is assumed to be given by the balancing mechanical production term (i.e. assuming local equilibrium)

$$
\epsilon=u_{*}^{2} \frac{d u}{d z} \propto u_{*}^{2} \frac{u_{*}}{\kappa z_{0 m}}
$$

For these assumptions, it directly follows that

$$
S h=A^{\prime} S c^{-1 / 2}\left(R e_{*}\right)^{-1 / 4}
$$

where $A^{\prime}$ is another similarity constant. Hence, the micro-eddy model (Brutsaert, 1965) predicts a $S h \propto R e_{*}^{-1 / 4}$. For most trace gases (or temperature), $S c$ (or the Prandtl number $P r$ ) is near unity in air and plays a lesser role (it was assumed unity in the LES here). Another important feature of the micro-eddy model is that the resulting $L_{i} \propto S c^{-1 / 2} \eta$ (i.e. the Batchelor length), where $\eta=\left(v^{3} / \epsilon\right)$ is, as before, equal to the Kolmogorov length scale. This scale is not resolved in the LES but is represented by the wall model. 


\section{3 | The macro-eddy model}

In this representation, the limiting step to heat or scalar exchange is assumed to be the efficiency of large coherent eddies to deliver parcels of air to the surface that then enable Kolmogorov eddies to exchange heat with the interface. The canopy sublayer air has a significantly higher concentration than the roughness sublayer air aloft, and surface renewal requires a large eddy to penetrate into, and ventilate, the canopy layer to sustain surface fluxes. Previous studies over large bluff-body obstacles (Leonardi et al., 2015) demonstrated that organized eddy motions indeed dominate in the roughness and canopy sublayers for turbulent scalar transport. For 'obstructed shear flows' (Ghisalberti, 2009) such as flows over urban canopies, the canopy flow can be characterized by the penetration depth $\left(L_{s}\right)$ of the vortices into the canopy sublayer. These can be linked to the shear length scale defined as $L_{s}=\frac{\bar{u}}{d \bar{u} / d z}$ for $z=H$ at the mean canopy height (Raupach et al., 1996). For most canopies, except the sparse ones, $L_{s} \leq H$ and in general $L_{s}$ is of the order of $H$ (Raupach et al., 1996; Katul et al., 1998; Poggi et al., 2004; Li and Bou-Zeid, 2019). The characteristic mean contact time scale can be related to the mean vorticity time scale, $(d \bar{u} / d z)^{-1}$ at $z=H$, which is $L_{s} / U_{H}$, where $U_{H}=\bar{u}(z=H)$. In addition, if the log-profile is extrapolated to $z=H, d \bar{u} / d z \approx u_{*} /\left(\kappa z_{0 m}\right)$. Indeed, the LES results depicted in Fig. 7a confirm that $(d \bar{u} / d z)^{-1}=L_{s} / U_{H}$ is correlated with $u_{*} / z_{0 m}$ (correlation coefficient is 0.73 with a $\mathrm{p}$-value $=0.001$ ). For this macro-eddy model, the characteristic mean contact time of renewal is proportional to the mean vorticity time scale (instead of the Kolmogorov time scale). Conceptually, this assumption implies that each single large coherent eddy leads to a single renewal event of the canopy sublayer air, and the number of renewal events must be identical to the frequency of occurrence of coherent events. Meanwhile, numerous other turbulent events, including Kolmogorov-sized eddies, renew the viscous sublayer multiple times but they are not the bottleneck in the renewal process (as their generation and delivery is dependent on the macro-eddy). This physical picture implies that large canopy-layer air ventilation events control the renewal rate, and that the entire building facet area then exchanges heat or scalars with each coherent eddy. Stated differently, the renewal eddy is 'space-filling' over a large contact area in this macro-eddy model. One can then contrast this to the micro-eddy model where numerous Kolmogorov eddies are assumed to be present at all points in the flow domain including all points around building facets.

Mindful of these physical assumptions, this macro-eddy model leads to

$$
\frac{1}{R} \sim \frac{L_{s}}{U_{H}} \sim \kappa \frac{z_{0 m}}{u_{*}}
$$

Now that the rate of surface renewal from macro eddies is estimated, it directly follows from Eq. 10 that

$$
S h=A^{\prime \prime} S c^{-1 / 2} R e_{*}^{-1 / 2}
$$

Therefore, the differing assumptions between the micro- and macro-eddy models result in distinct scaling laws between $S h$ and $R e_{*}$.

\subsection{The chain-saw model}

This model was originally formulated to explore air-water gas exchange in streams (Moog and Jirka, 1999). The basic assumptions can be contrasted to the urban roughness exchange mechanisms discussed here. In its original form, surface renewal is analogous to a chain-saw cutting a tree. The small teeth of the chain-saw perform the actual cutting (much like the Kolmogorov eddies cutting through the viscous sublayer in the micro-eddy approach) but the 
chain speed and the larger saw blade (much like the large and coherent eddies) carry the small-teeth to the cutting zone (the viscous sublayer attached to solid boundaries). Both the speed of the chain and the access of the small blades to the cutting zone control the actual cutting. Both scales 'cooperate' and can now be incorporated for urban roughness by adopting the active zones approach of the chain saw model. In this case, each eddy penetration encounters an incomplete interfacial area not covering the entire surface area. The chain-saw model then assumes that within each patch area covered by the big eddy, Kolmogorov (or Batchelor) sized eddies carry out the scalar exchange as in the micro-eddy model. The micro-eddy model still represent the correct physics but must be amended to include the 'active zone' fractional area $A_{a}^{+}$reflecting the penetration and renewal from large eddies. It is straightforward to show that this representation leads to

$$
S h=A^{\prime} S c^{-1 / 2}\left(A_{a}^{+}\right)\left(R e_{*}\right)^{-1 / 4},
$$

where $A_{a}^{+}$is now expected to scale with $R e_{*}$ and the arrangement of the roughness elements. If $A_{a}^{+} \sim R e_{*}^{0}$, then the micro-eddy model is restored, whereas with $A_{a}^{+} \sim R e_{*}^{-1 / 4}$, the macro-eddy model is recovered.

In the chain-saw model, at a fixed $S h$ and for an imposed $u_{*}$ dictating the energy available for eddy penetration into the canopy elements (set by $u_{*}^{2}$ ), increasing $z_{0 m}$ increases $R \boldsymbol{e}_{*}$ but reduces the eddy penetration and hence $A_{a}^{+}$. The chain-saw model is featured here not because of its prognostic skills - but its diagnostic skills. It suggests that a number of possible exponents are possible depending on how efficient the eddy penetration into the cuboids is. Another amendment to the chain-saw model may include the spatial intermittency of $\epsilon$ at the walls of the cuboids for each eddy penetration, adding another relation between $R e_{*}$ and $\epsilon$. The scaling of $A_{a}^{+}$with $R e_{*}$ is rarely studied in urban canopies and must be externally supplied for now. Nonetheless, the analysis here provides plausible explanations as to why differing scaling exponents for $S c-R e_{*}$ may have been reported in the literature.

\section{5 | Linking Surface Renewal to $K B^{-1}$}

As noted, the $S h$ derived from SRT can be interpreted as both an interfacial and bulk characteristic extending to the inertial layer. If so, $S h$ can then be determined from equation 2 in the neutral limit as

$$
S h^{-1}=\frac{1}{\kappa} \log \left(\frac{z-d_{s}}{z_{0 s}}\right) \text {. }
$$

Similarly, the interfacial bulk drag coefficient for the virtual bluff-rough surface $C_{m}$, Eq. 6 , can be related to $z_{0 m}$ as

$$
C_{m}^{-1 / 2}=\frac{1}{\kappa} \log \left(\frac{z-d}{z_{0 m}}\right) .
$$

Then, assuming $d=d_{s}, z_{0 m}$ and $z_{0 s}$ can be related using $S h$ and $C_{m}$ using

$$
z_{0 s}=z_{0 m} \exp \left[-\kappa\left(S h^{-1}-C_{m}^{-1 / 2}\right)\right]
$$

in which $S h^{-1}-C_{m}^{-1 / 2}$ is usually referred to as $B^{-1}$, i.e. $z_{0 s}=z_{0 m} \exp \left[-\kappa B^{-1}\right]$. For the macro-eddy model, the $B^{-1}$ can be expressed as:

$$
B^{-1}=S h^{-1}-C_{m}^{-1 / 2}=A_{1} R e_{*}^{1 / 2}-C_{m}^{-1 / 2}=A_{1} R e_{*}^{1 / 2}+A_{2}
$$


where $A_{1}$ is $1 / A^{\prime}$, and $A_{2}=-C_{m}^{-1 / 2}$. In the fully rough limit at high $R e, C_{m}$ only depends on the geometry of the surface and should become Reynolds-number independent (equivalent to stating that $d$ and $z_{0 m}$ are independent of wind speed and fluid viscosity). It is also worth noting that the $A_{2}$ parameter obtained as the intercept from field data using statistical regression (Kanda et al., 2007) has a clear physical meaning that pertains to the bulk drag coefficient as $A_{2}=-C_{m}^{-1 / 2}$, and it is constant only under the assumption of $C_{m}$ being a much weaker function of $R e_{*}$ than $S h$.

Fig. 7a shows the calculated $B^{-1}$ from LES for all the three-dimensional rough surfaces in Table 1; models from Brutsaert $(1965,1975 a)$ and Kanda et al. (2007) are also shown. The model by Brutsaert (1965, 1975a) is based on the micro-eddy model and results in $B^{-1}=C_{1} R e_{*}^{1 / 4}+C_{2}$ instead of $R e_{*}^{1 / 2}$ for the macro-eddy model. The model by Kanda et al. (2007) assumes the same one-fourth power relation between $B^{-1}$ and $R e_{*}$. These models with an exponent $\approx 1 / 4$ do not seem to be the most appropriate for the large bluff roughness elements of this study despite the fact that they were reported to match observations better (than ones with exponents $\approx 1 / 2$ ) over natural surfaces such as waves (Li et al., 2018). We could improve these models by refitting their constants to the LES data, but given the mismatch in the slope this was deemed unnecessary. The macro-eddy model captures the LES derived $B^{-1}-R e_{*}$ scaling better. Additional relevant models in the literature are shown in Table 2. Zilitinkevich et al. (2001) obtained $R e_{*}^{1 / 2}$ scaling, but the result was derived based on dimensional analysis without invoking surface renewal theory (yet it matches the macro-eddy model in scaling).

(a)

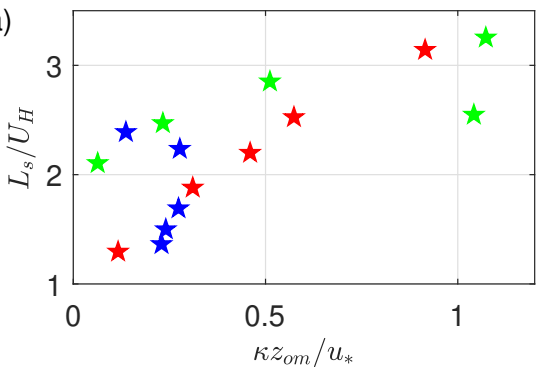

(b)

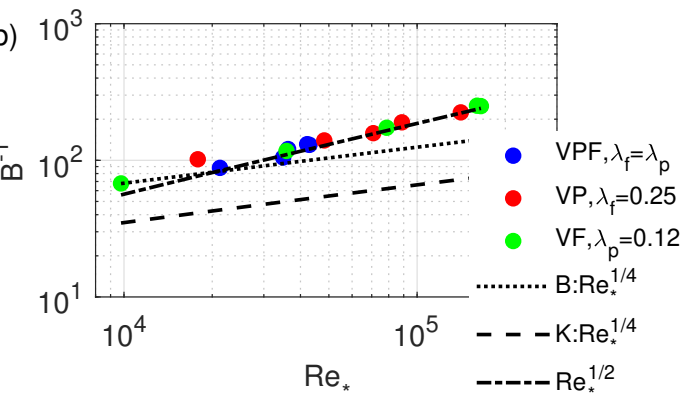

FIGURE 7 (a) The relation between $L_{s} / U_{H}$ and $k z_{0 m} / u_{*}$ showing reasonably-linear trends. The macro-eddy model assumes that the mean contact time $1 / R$ can be estimated as $k z_{0 m} / u_{*}$. (b) $B^{-1}$ as a function of $R e_{*}=u_{*} z_{0 m} / v$ from LES and various models. The SRT predictions from the micro-eddy and macro-eddy models are also shown. B: $R e_{*}^{1 / 4}$ Brutsaert (1975b) model: $7.3 S c^{1 / 2} R e_{*}^{1 / 4}-5$. $\mathrm{K}: R e_{*}^{1 / 4}$ Kanda et al. (2007) model: $6.4 R e_{*}^{1 / 4}-5$. $R e_{*}^{1 / 2}$ least-square regression of the macro-eddy power law model: $B^{-1}=S h^{-1}-C_{m}^{-1 / 2}=0.60 R e_{*}^{1 / 2}-3.5$.

A least-square regression with $R e_{*}^{1 / 2}$ yields the constants $A_{1}=0.68$ and $A_{2}=3.5$ (Table 2, Method 1). Leastsquare fitting of $S h^{-1} \propto R e_{*}^{1 / 2}$ and $S h^{-1} \propto R e_{*}^{1 / 4}$ found that the 1/2 scaling yielded a coefficient of determination of 0.96 (cf. 0.80 for the $1 / 4$ scaling). This finding confirms that the SRT with the macro-eddy representation is consistent with the wall-modeled LES computations of macro-scale scalar roughness length. It should also be noted that $B^{-1}$ versus $R e_{*}$ can have a large range of variability (cf. Figure 6 in Kanda et al. (2007), Figure 2 in Crawford et al. (2018)), which can be attributed to variability in the multiplicative factor $A_{1}$ or from other physical processes not captured here that could also alter the power exponent relating $B^{-1}$ and $R e_{*}$.

The functional dependence between $S h^{-1}$ and $R e_{*}$ (Fig. 8) can now be exploited to propose a new approach to parameterize $z_{0 s}$ for urban terrain. Morphometric models, (e.g. Kanda et al. (2013) can be used to obtain $z_{0 m}$ and $d$, and therefore compute $C_{m}^{-1 / 2}$, according to Eq. 18 for a given z). Using Eq. 20 with $A_{1}=0.70$ deduced from LES 
here, (or fit other observational or LES data for other distinct geometries), $z_{0 s}$ results are obtained (Table 2, Method 2). Direct evaluation of $z_{0 s}$ obtained from LES in Fig.8b (we compare the logarithms since they are the forms that appear in conventional models) have good agreement.

\begin{tabular}{lc} 
Reference & $B^{-1}$ \\
\hline Owen and Thomson (1963) & $2.40 R e_{*}^{0.45}$ \\
Brutsaert (1975a) & $7.3 S c^{1 / 2} R e_{*}^{1 / 4}-5$ \\
\hline Cahill et al. (1997) & $4.31 R e_{*}^{1 / 4}-5$ \\
\hline Zilitinkevich et al. (2001) & $0.5 R e_{*}^{1 / 2}-3.2$ \\
\hline Method 1: Eq. 20 with constant A2 & $0.60 R e_{*}^{1 / 2}-3.5$ \\
Method 2: Eq. 20 with variable $A_{2}=-C_{m}^{-1 / 2}$ from morphometric model & $0.70 R e_{*}^{1 / 2}-C_{m}^{-1 / 2}$
\end{tabular}

TABLE 2 Relation between $B^{-1}$ and roughness Reynolds number $R e_{*}$ from different studies.
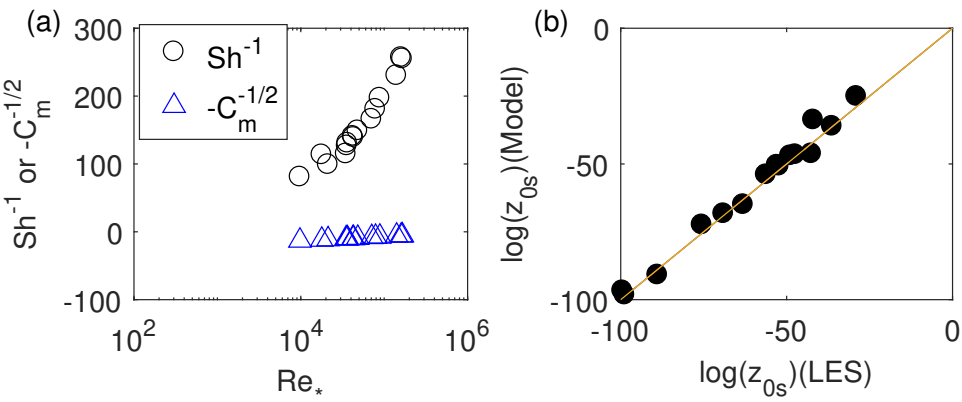

FIGURE 8 Variations of $S h^{-1}$ and $C_{m}^{-1 / 2}$ with $R e_{*}$ (a) and scalar roughness length $z_{0 s}$ modelled (Method 2, Table 2) versus results directly from LES.

\section{5 | CONCLUSIONS}

Large-eddy simulations for urban surfaces consisting of arrays of large cuboids are conducted to address how urban roughness elements impact the variations of scalar roughness length when compared to its momentum counterpart. The simulations had frontal area density $\lambda_{f}$ and plan area density $\lambda_{p}$ varied independently. The variations in $z_{0 m}$ are linked to the combination of increasing with $\lambda_{f}$ and decreasing with $\lambda_{p}$. Models based on morphometric parameters have acceptable predictive power to capture these trends for $z_{0 m}$. To link such models for $z_{0 m}$ to $z_{0 s}$, the $\kappa B^{-1}=$ $\log \left(z_{0 m} / z_{0 s}\right)=A_{1} R e_{*}^{n}+A_{2}$ framework was used. An approach based on the surface renewal theory is proposed to determine $n$. The exponent derived here closely follows its theoretical prediction when the mean contact time is derived from macro-eddy properties $(n=1 / 2)$ instead of micro-eddy properties $(n=1 / 4)$. An alternative model that is based on the chain-saw analogy was also explored. This model features an effective contact area depending on eddy penetration, and can recover either the micro- or macro-eddy models scaling, depending on how this area scales with 
$R e_{*}$.

The results here have three major implications:

(i) There are "trade-off effects" in the effectiveness of a very-rough surface in exchanging momentum and scalars with a turbulent flow as can be deduced from the opposing trends of $C_{m}$ and $C_{h}$ with changes in geometry. Similar findings have been reported over steep waves (Sullivan et al., 2017). These findings illustrate the contrasting effects of roughness elements, as discussed by Brutsaert (1975b) and others: "... (roughness elements) on the one hand, they act as windbreaks, reducing surface exposure and thus transfer, while on the other hand, they increase drag and thus shear with a concomitant increase in eddy diffusivity". Roughness elements are usually applied to a smooth wall to increase turbulence mixing, which enhances both drag and heat transfer. For urban surfaces, the results here imply that as the density of roughness elements increases, a point will be reached beyond which convective heat transfer will become less efficient. Similarly, skin hairs promote heat transfer at low densities but acts as insulators at higher densities (Fowler and Bejan, 1995).

(ii) In contrast to land surface models that postulate a relation $z_{0 s} \propto z_{0 m}$ (usually with a pre-factor of 1/10), the LES predicts that an increase in $z_{0 m}$ results in a decrease $z_{0 s}$. In addition, the macro-eddy SRT model proposed in this paper, as well as all other models evaluated here (including those closely following the micro-eddy SRT) also predict an inverse relation of the two roughness lengths (for typical values of the friction velocity, air viscosity and momentum roughness length in urban terrain; Fig. 9 a with a friction velocity $u_{*}=1 \mathrm{~m} \mathrm{~s}^{-1}$ ). Different values $u_{*}$ values dictate changes in $z_{0 s}$ as $z_{0 m}$ varies, which gives rise to the scatter in LES results shown in Fig. 9b. Data from other studies are also shown in Fig.9b. Despite the large range of uncertainty, that $z_{0 s}$ decreases with increasing $z_{0 m}$ is consistent with these data.
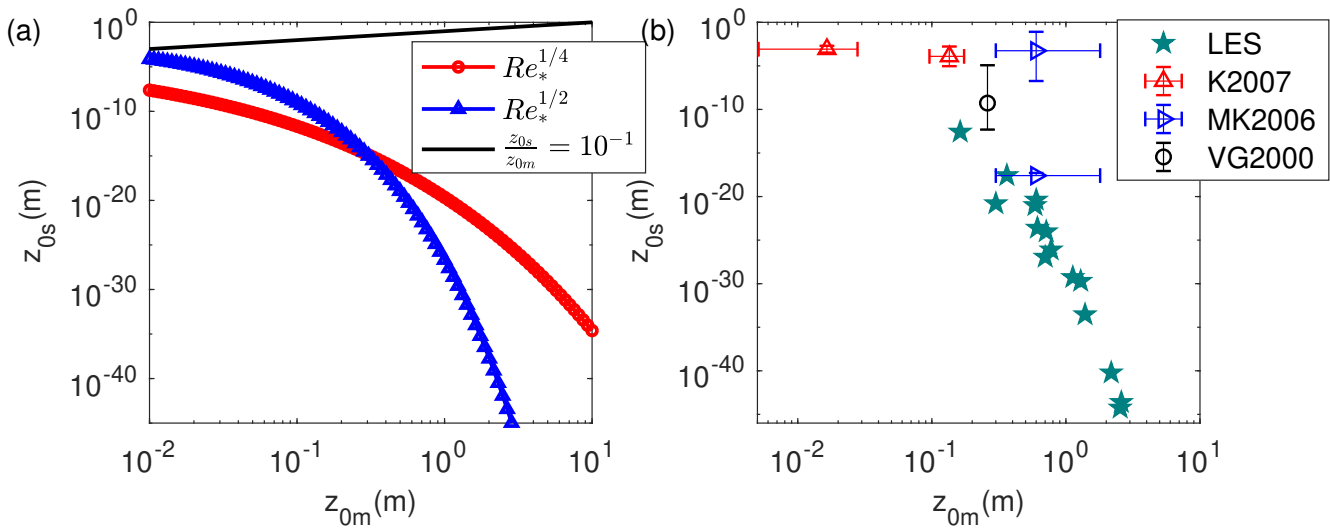

FIGURE 9 (a): Variation of $z_{0 s}$ with $z_{0 m}$ for a typical friction velocity $u_{*}=1 \mathrm{~m} / \mathrm{s}, v=1.46 \times 10^{-5} \mathrm{~m}^{2} / \mathrm{s}$. $z_{0 s}$ modeled with Brutsaert (1975a) (red circle); $z_{0 s}$ modeled with Method 1 in this study (blue triangle); $z_{0 s} / z_{0 m}=10^{-3}$ (black line). (b): LES and previous field measurements. LES (teal stars); K2007 (red triangle): outdoor urban scale models in Kanda et al. (2007), the whiskers indicate standard deviations of momentum and heat roughness lengths reported in Table 3 of Kanda et al. (2007); MK2006 (blue triangle): measurements from a suburban site in Tokyo, Japan by Moriwaki and Kanda (2006b) showing momentum, heat (upper triangle) and water vapor (lower triangle) roughness lengths reported in Table 1 of Moriwaki and Kanda (2006b), where the whiskers indicate their respective standard deviations; VG2000 (black circle): measurements from a light industrial site in Vancouver, British Columbia, Canada from (Voogt and Grimmond, 2000). Data are extracted from their Fig.8a, where the whiskers indicate the range of heat roughness lengths. 
(iii) As obstacle-resolving LES is becoming a promising tool to explore urban atmospheric boundary layers. However, how to interpret the bulk transfer properties directly computed from LES, especially for scalars, remains a subject of inquiry. The values that emerge from the analysis can depend on the wall-model used to link the obstacle surface to the flow. The wall-model formulation used here is self-consistent with expected theoretical behavior of an aerodynamically smooth wall, as well as the micro-eddies in the surface renewal theory (Appendix B).

(iv) The feasibility of estimating $z_{0 s}$ for urban surfaces using a two-step approach is demonstrated. This should be verified, using field observations and/or LES or DNS, for other more realistic geometries. This method utilizes the $R e_{*}$ dependence of scalar transfer and the adequate skill of the existing morphometric momentum roughness length models. Here only Macdonald et al. (1998) is used to illustrate this two-step approach to compute $C_{m}$. Future studies could use newer models (e.g. (Kanda et al., 2013; Yang et al., 2016; Zhu and Anderson, 2018), etc.) for $d$ and $z_{0 m}$ to further refine $C_{m}$.

Although the number of cases with idealized cuboids is limited, the generalized framework of both micro- and macro-scale roughness lengths can be extended and tested for other configurations (e.g. a larger morphometric parameter space and realistic urban geometries). In addition, the impact of different thermal boundary conditions and atmospheric stability regimes have been purposely omitted, but these effects are expected to have a significant influence on $d, z_{0 m}$, and $z_{0 s}$. Last, the 'chain-saw' model for surface renewal opened up the inquiry into how to connect the penetration of eddies into the roughness elements with $R \boldsymbol{e}_{*}$ and whether the turbulent kinetic energy dissipation rate is spatially intermittent with patchy contact wall areas that scale with $R e_{*}$. Recent advances in thermal infrared imaging may offer some clues on how to proceed; this is also a topic that can be explored using DNS in future studies.

\section{Appendix A: Numerical setup}

The LES model used here implements the immersed boundary method (IBM) to resolve the bluff-body obstacles explicitly. The non-dimensional filtered incompressible continuity (A 1), Navier-Stokes (A 2) and scalar conservation (A 3) equations are solved assuming hydrostatic equilibrium of the mean flow:

$$
\frac{\partial u_{i}}{\partial x_{i}}=0
$$

$$
\frac{\partial u_{i}}{\partial t}+u_{j}\left(\frac{\partial u_{i}}{\partial x_{j}}-\frac{\partial u_{j}}{\partial x_{i}}\right)=-\frac{\partial p}{\partial x_{i}}-\frac{\partial \tau_{i j}}{\partial x_{j}}+F_{i}+B_{i}
$$

$$
\frac{\partial \theta}{\partial t}+u_{i} \frac{\partial \theta}{\partial x_{i}}=-\frac{\partial q_{i}^{s}}{\partial x_{i}}
$$

where $x, y$ and $z$ denote the streamwise, cross-stream and wall-normal directions respectively, and $u, v$ and $w$ are the velocity components in these respective directions; $t$ denotes time; $u_{i}$ is the resolved velocity vector; $p$ is the modified pressure; $\tau_{i j}$ is the deviatoric part of the subgrid stress tensor; $F_{i}$ is the body force driving the flow (here simply a homogeneous steady horizontal pressure gradient imposed along the $x$ direction); and $B_{i}$ is the immersed boundary force representing the action of the obstacles on the fluid. In equation (A 3), $\theta$ denotes the concentration of a passive scalar quantity and $q_{i}^{s}$ is the $i^{\text {th }}$ component of the subgrid scale scalar flux. All the variables used are the filtered components, although the usual tilde on top is omitted for notational simplicity. Equations A 1 to A 3 are prenormalized by the friction velocity that accounts for the total (viscous and form) surface drag $\left(u_{*}\right)$, the boundary-layer depth $(\delta)$, air density $(\rho)$ and a reference scalar quantity $\left(\theta_{0}\right)$. 
The passive scalar $\theta$ considered here is temperature, but the buoyancy force is omitted to mimic a passive scalar (the gravitational acceleration is set to zero); the results would be applicable to all other nearly-passive scalars such as water vapor. However, future extension to include buoyant forces under strong heating is required to proceed towards realistic conditions. An isothermal boundary condition of $330 \mathrm{~K}$ is imposed on all surfaces. Horizontal periodic boundary conditions are applied to all quantities. The top boundary conditions are zero stress and scalar flux and no-penetration (zero normal velocity). Further details regarding the numerical procedures are described elsewhere (Chester et al., 2007; Li et al., 2016a) and the subgrid scale model is discussed in Bou-Zeid et al. (2005). The code has been verified (Li et al., 2016a,b) for its performance in simulating both momentum- and scalar- transport. All simulations are run for 50 eddy turn-over times defined as $\delta / u_{*}$ and the last 25 are averaged for the statistics analyzed here.

\section{Appendix B: The relation between wall-modeled $F_{s}$ and $z_{0 s}^{m i c}$}

Implications of micro-scale roughness values in the LES wall-model in terms of $F_{s}$ (the scalar fluxes computed from the wall model) are considered. The surface renewal theory (Brutsaert, 1982) for surfaces that are aerodynamically smooth or having intermediate roughness elements indicate a sub-linear dependence on friction velocity $\left(\propto u_{*}^{3 / 4}\right)$ of surface fluxes of passive admixtures. Such a sub-linear dependence discussed elsewhere (Katul and Liu, 2017) has been shown not to depend on the assumptions intrinsic to surface renewal theory. For example, the mean flux of a passive scalar admixture (e.g. water vapor) (Katul and Liu, 2017) is :

$$
F_{s} \propto D_{m}^{1 / 2} u_{*}^{3 / 4}\left(\kappa v z_{0 m}^{m i c}\right)^{-1 / 4}\left(\theta_{s}-\theta_{h}\right),
$$

where $D_{m}$ is molecular diffusivity of water vapor; $\theta_{s}$ and $\theta_{h}$ are mean scalar concentration (or temperature) at the surface and interfacial sublayer, respectively. Physically, the micro-scale roughness length is a bulk representation of the thickness of the interfacial sublayer and of (i) either the molecular processes therein for smooth walls, or (ii) of the roughness geometries of the surface for intermediate rough walls. In the context of the LES with smooth walls here, $z_{0 m}^{m i c}$ is given by the wall-model (Eq. 3) and also depends on $u_{*}$. If we substitute the wall-model into equation B 1 , the resulting wall-modeled scalar flux $F_{s}$ follows

$$
F_{s} \propto\left(\frac{9}{\kappa}\right)^{1 / 4} S c^{-1 / 2} u_{*}^{w m}\left(\theta_{s}-\theta_{h}\right)
$$

where $S c=v / D_{m}$ is the molecular Schmidt number; $\theta_{h}$ is the mean scalar magnitude in the vicinity of the surface.

By virtue of the wall model here, where $S c=1$, Eq. B 2 becomes

$$
F_{s} \propto u_{*}^{w m}\left(\theta_{s}-\theta_{h}\right)
$$

Figure B 1 shows $F_{s}$ versus $u_{*}^{w m}$ and the least-square regression using $u_{*}^{w m}$ computed from all facets for all cases in Table 1. The data from LES suggest that $F_{s}$ practically follows a linear scaling with $u_{*}^{w m}$, (i.e. the power exponent is $1.005 \approx 1.0$ ), which confirms that imposing a smooth-surface micro-scale roughness length in the wall model for LES is consistent with the prediction in Eq. B 3 and the general surface renewal theory scaling when a smooth-wall roughness length is desired. In fact, Eq. B 3 or Eq. 11 are more generalizable results since surface renewal theory at the micro-scale does not need to be invoked to obtain these relations (Katul and Liu, 2017). Imposing a wall function in LES based on the log-law formulation implicitly satisfies the assumptions of Kolmolgorov's inertial subrange scaling 
(Gioia et al., 2010). This finding suggests that the results obtained from LES wall-models are not dependent on the validity of surface renewal theory at the micro-scale. The analysis here is intended to show self-consistency of the wall-modeling approach adopted here.

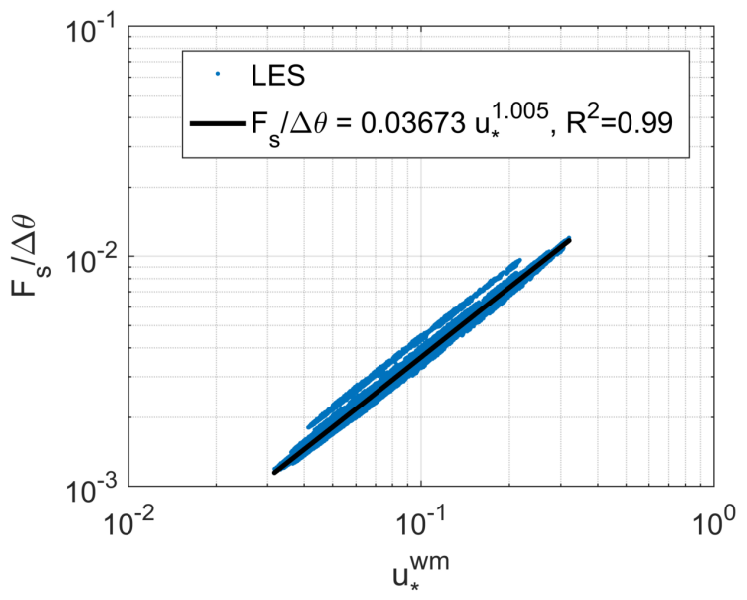

FIGURE B $1 F_{s} /\left(\theta_{s}-\theta_{h}\right)$ versus $u_{*}^{w m}$ for all LES runs. Each data point represents the mean $F_{s}$ and $u_{*}^{w m}$ obtained from the wall model.

\section{Appendix C: Uncertainty Analysis in the Delineation of the Log-Region on Macro-Roughness Parameters}

The delineation of the logarithmic region affects the zero-plane displacement and roughness length estimation is explored using least-squares regression fitting over all plausible ranges in the logarithmic region (from just above the building height to three times the building height by visual inspection of the profiles), using at least six points. A relative sensitivity factor is defined for each fitted parameter $Y$ as $Y-Y_{0} /\left(Y_{\max }-Y_{\min }\right)$, where $Y_{0}$ is the value for the range of fitting adopted in the paper (i.e. logarithmic region starts from $1.25 \mathrm{H}$ and ends at $2.25 \mathrm{H}$ ); $Y$ is the value of a given range; $Y_{\max }(\min )$ is the maximum (minimum) value of $Y$ obtained for all possible ranges. The parameters tested are $z_{0 m}, z_{0 s}$ or $d$, and their sensitivity plots are shown in Fig.C 1, Fig.C 2 and Fig. C 3, respectively.

The relative sensitivity factor, $D_{v}$, averaged for each geometry over all plausible ranges of the log-region in which the least-square fitting is performed, is always less than about $40 \%$, and it is within $20 \%$ for most of the cases (Fig. C 4). Therefore, the sensitivity of results to the precise delineation of the logarithmic regions does not change the overall trends in $B^{-1}=\log \left(z_{0 m} / z_{0 s}\right)$ as shown in Fig. C 5). The conclusions of the paper are deemed robust to uncertainties in the identification of the log-region.

\section{references}

Anderson, W. (2013) Passive scalar roughness lengths for atmospheric boundary layer flow over complex, fractal topographies. Environmental Fluid Mechanics, 13, 479-501.

Barlow, J. F. and Coceal, O. (2009) A review of urban roughness sublayer turbulence. Met Office Research and DevelopmentTechnical Report, 1, 527. 

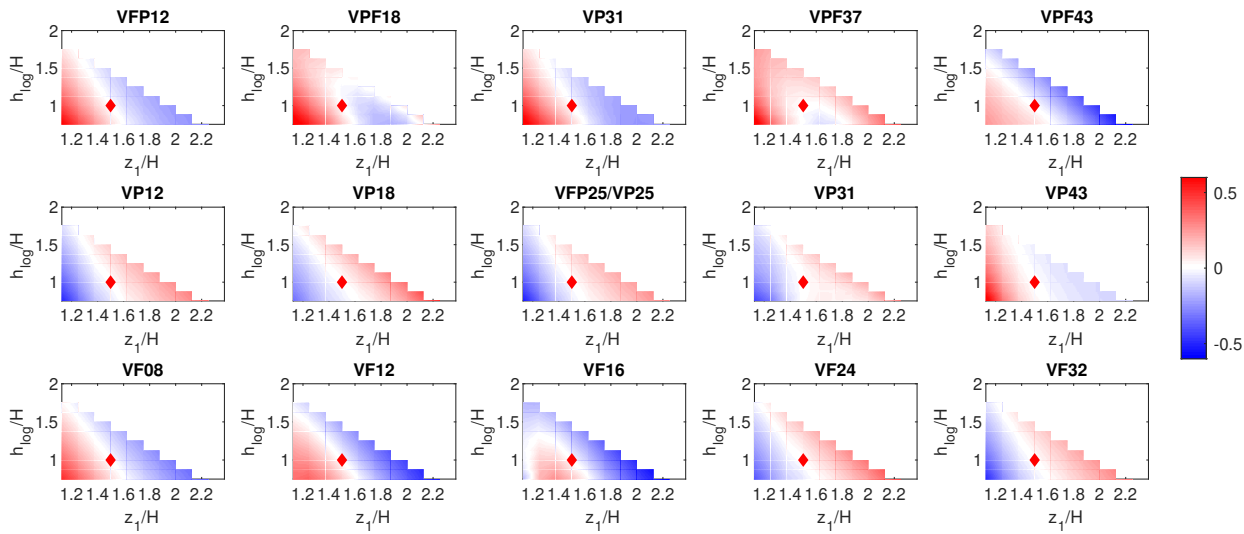

FIGURE C 1 The range of variability of $z_{0 m}$ obtained by varying $z_{1} / H$, the position where the log-layer commences, and the span of the log-region, $h_{\log } / H$. The red diamond shape denotes the chosen range of the log-region for least-square regression featured in the main text (i.e. $z_{1} / H=1.25$ and $h_{\log } / H=1$ ). Colorbar indicates the relative sensitivity factor.
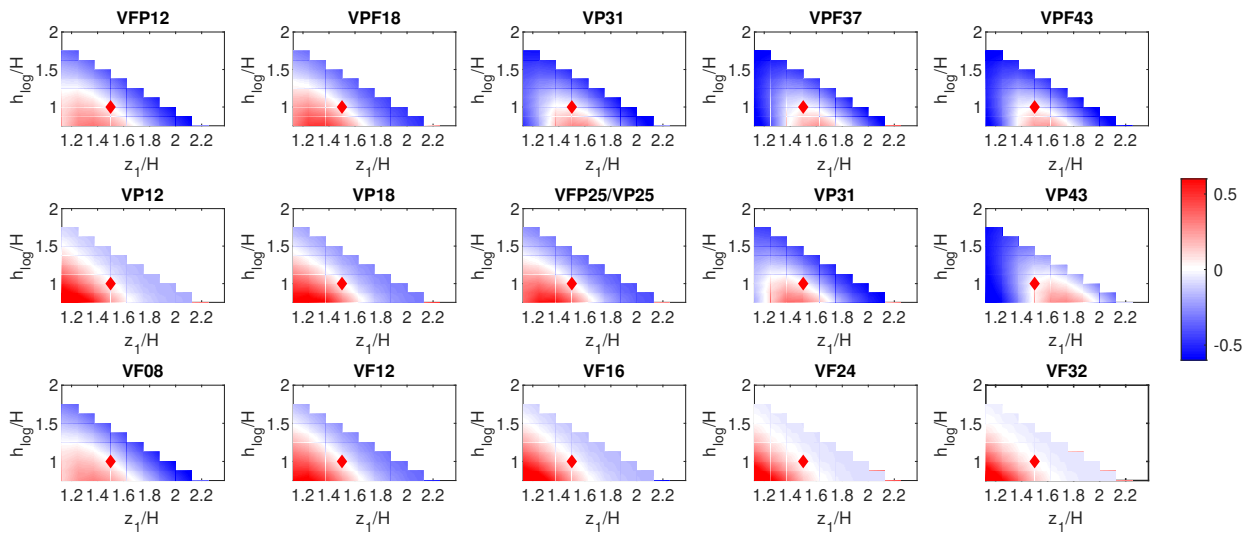

FIGURE C 2 As Fig.C1, but for $z_{0 s}$. 

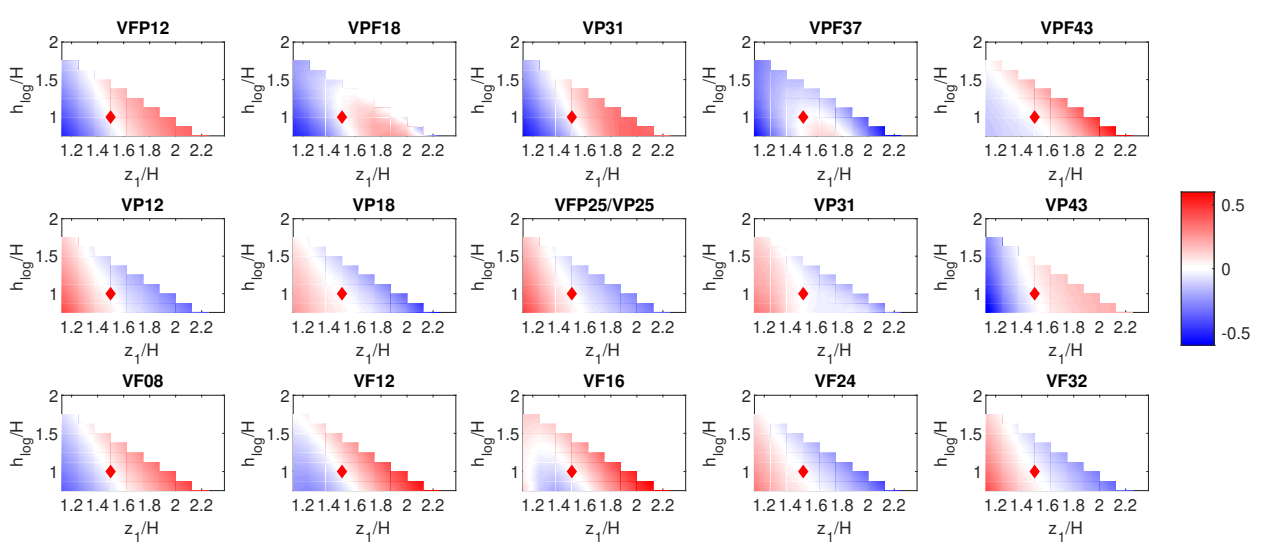

FIGURE C 3 As Fig.C1, but for $d$.

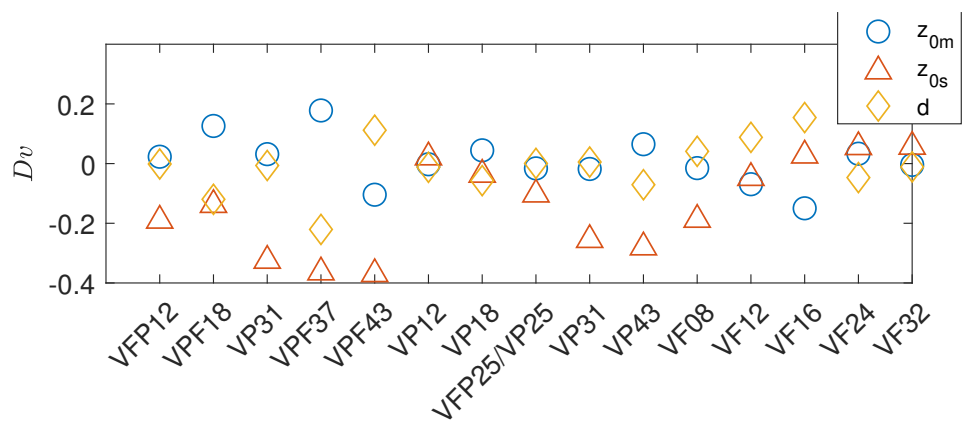

FIGURE C 4 Averaged sensitivity factor $D v$ for $z_{0 m}, z_{0 s}$ and $d$.

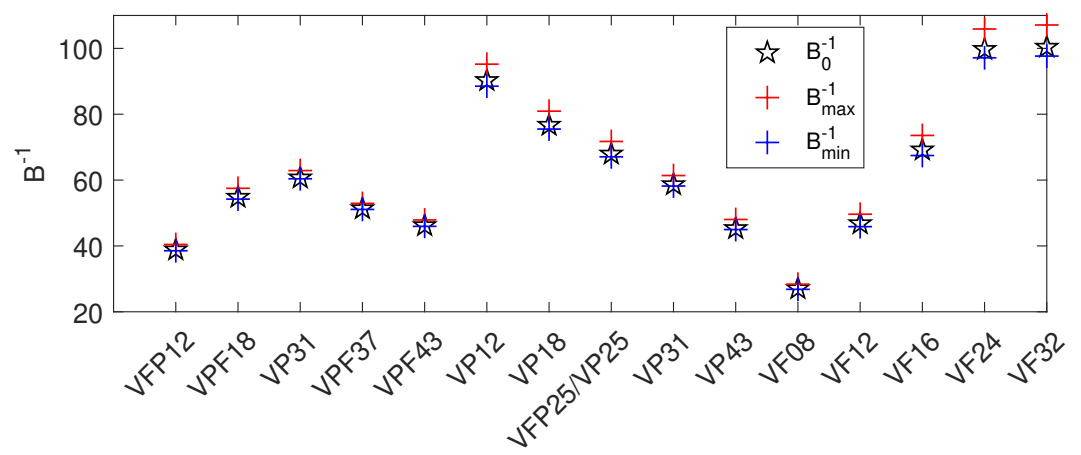

FIGURE C 5 Variation of $B^{-1}$ across cases. $B_{0}^{-1}$ is computed from $z_{0 m}$ and $z_{0 s}$ obtained for $z_{1} / H=1.25$ and $h_{\log } / H=1 ; B_{\max }^{-1}$ and $B_{\min }^{-1}$ are obtained from the maximum and minimum values of $z_{0 m} / z_{0 s}$ for all values from the possible ranges of logarithmic region. 
Barlow, J. F., Harman, I. N. and Belcher, S. E. (2004) Scalar fluxes from urban street canyons. Part I: Laboratory simulation. Boundary-Layer Meteorology, 113, 369-385.

Bose, S. T. and Park, G. I. (2018) Wall-modeled large-eddy simulation for complex turbulent flows. Annual Review of Fluid Mechanics, 50, 535-561.

Bou-Zeid, E., Meneveau, C. and Parlange, M. (2005) A scale-dependent Lagrangian dynamic model for large eddy simulation of complex turbulent flows. Physics of Fluids, 17, 1-18.

Bou-Zeid, E., Overney, J., Rogers, B. D. and Parlange, M. B. (2009) The effects of building representation and clustering in large-eddy simulations of flows in urban canopies. Boundary-Layer Meteorology, 132, 415-436.

Brutsaert, W. (1965) A model for evaporation as a molecular diffusion process into a turbulent atmosphere. Journal of Geophysical Research, 70, 5017.

- (1975a) A theory for local evaporation (or heat transfer) from rough and smooth surfaces at ground level. Water Resources Research, 11, 543-550.

- (1975b) The roughness length for water vapor sensible heat, and other scalars. Journal of Atmospheric Sciences, 32, 20282031.

- (1982) Evaporation into the atmosphere: theory, history and applications, vol. 1. Springer Science \& Business Media.

Cai, X.-M. (2012) Effects of wall heating on flow characteristics in a street canyon. Boundary-Layer Meteorology, 142, $443-467$.

Castellví, F. (2018) An advanced method based on surface renewal theory to estimate the friction velocity and the surface heat flux. Water Resources Research, 54, 10-134.

Castellvi, F., Snyder, R. and Baldocchi, D. (2008) Surface energy-balance closure over rangeland grass using the eddy covariance method and surface renewal analysis. Agricultural and Forest Meteorology, 148, 1147-1160.

Chen, F. and Zhang, Y. (2009) On the coupling strength between the land surface and the atmosphere: From viewpoint of surface exchange coefficients. Geophysical Research Letters, 36.

Chen, Y., Yang, K., Zhou, D., Qin, J. and Guo, X. (2010) Improving the noah land surface model in arid regions with an appropriate parameterization of the thermal roughness length. Journal of Hydrometeorology, 11, 995-1006.

Chester, S., Meneveau, C. and Parlange, M. B. (2007) Modeling turbulent flow over fractal trees with renormalized numerical simulation. Journal of Computational Physics, 225, 427-448.

Chung, J., Hagishima, A., Ikegaya, N. and Tanimoto, J. (2015) Wind-Tunnel Study of Scalar Transfer Phenomena for Surfaces of Block Arrays and Smooth Walls with Dry Patches. Boundary-Layer Meteorology, 157, 219-236.

Clayson, C. A., Fairall, C. W. and Curry, J. A. (1996) Evaluation of turbulent fluxes at the ocean surface using surface renewal theory. Journal of Geophysical Research: Oceans, 101, 28503.

Crawford, B., Grimmond, S. B., Gabey, A., Marconcini, M., Ward, H. C. and Kent, C. W. (2018) Variability of urban surface temperatures and implications for aerodynamic energy exchange in unstable conditions. Quarterly Journal of the Royal Meteorological Society, 144, 1719-1741.

Danckwerts, P. V. (1951) Significance of liquid-film coefficients in gas absorption. Industrial \& Engineering Chemistry, 43, 14601467.

De Ridder, K. (2006) Testing brutsaert's temperature roughness parameterization for representing urban surfaces in atmospheric models. Geophysical Research Letters, 33. 
Demuzere, M., De Ridder, K. and Van Lipzig, N. (2008) Modeling the energy balance in marseille: Sensitivity to roughness length parameterizations and thermal admittance. Journal of Geophysical Research: Atmospheres, 113.

Denby, B. and Snellen, H. (2002) A comparison of surface renewal theory with the observed roughness length for temperature on a melting glacier surface. Boundary-Layer Meteorology, 103, 459-468.

Flack, K. A. and Schultz, M. P. (2014) Roughness effects on wall-bounded turbulent flows. Physics of Fluids, 26.

Fowler, A. J. and Bejan, A. (1995) Forced convection from a surface covered with flexible fibers. International journal of heat and mass transfer, 38, 767-777.

Garratt, J. (1980) Surface influence upon vertical profiles in the atmospheric near-surface layer. Quarterly Journal of the Royal Meteorological Society, 106, 803-819.

Garratt, J. R. (1992) The atmospheric boundary layer, cambridge atmos. Space Sci. Ser., Cambridge Univ. Press, Cambridge, UK.

Ghisalberti, M. (2009) Obstructed shear flows: similarities across systems and scales. Journal of Fluid Mechanics, 641, 51-61.

Gioia, G., Guttenberg, N., Goldenfeld, N. and Chakraborty, P. (2010) Spectral theory of the turbulent mean-velocity profile. Physical review letters, 105, 184501.

Giometto, M. G., Christen, A., Meneveau, C., Fang, J., Krafczyk, M. and Parlange, M. B. (2016) Spatial Characteristics of Roughness Sublayer Mean Flow and Turbulence Over a Realistic Urban Surface. Boundary-Layer Meteorology, 1-28.

Grimmond, C. and Oke, T. R. (1999) Aerodynamic properties of urban areas derived from analysis of surface form. Journal of Applied Meteorology.

- (2002) Turbulent heat fluxes in urban areas: Observations and a local-scale urban meteorological parameterization scheme (lumps). Journal of Applied Meteorology, 41, 792-810.

Hagishima, A., Tanimoto, J., Nagayama, K. and Meno, S. (2009) Aerodynamic parameters of regular arrays of rectangular blocks with various geometries. Boundary-Layer Meteorology, 132, 315-337.

Harman, I. N. and Finnigan, J. J. (2007) A simple unified theory for flow in the canopy and roughness sublayer. Boundary-Layer Meteorology, 123, 339-363.

- (2008) Scalar concentration profiles in the canopy and roughness sublayer. Boundary-Layer Meteorology, 129, 323-351.

Ikegaya, N., Hagishima, A., Tanimoto, J., Tanaka, Y., Narita, K. and Zaki, S. A. (2012) Geometric Dependence of the Scalar Transfer Efficiency over Rough Surfaces. Boundary-Layer Meteorology, 143, 357-377.

Jackson, P. S. (1981) On the displacement height in the logarithmic velocity profile. Journal of Fluid Mechanics, 111, 15.

Kader, B. and Yaglom, A. (1972) Heat and mass transfer laws for fully turbulent wall flows. International Journal of Heat and Mass Transfer, 15, 2329-2351.

Kanda, M., Inagaki, A., Miyamoto, T., Gryschka, M. and Raasch, S. (2013) A new aerodynamic parametrization for real urban surfaces. Boundary-Layer Meteorology, 148, 357-377.

Kanda, M., Kanega, M., Kawai, T., Moriwaki, R. and Sugawara, H. (2007) Roughness lengths for momentum and heat derived from outdoor urban scale models. Journal of Applied Meteorology and Climatology, 46, 1067-1079.

Kanda, M., Moriwaki, R. and Kasamatsu, F. (2004) Large-eddy simulation of turbulent organized structures within and above explicitly resolved cube arrays. Boundary-Layer Meteorology, 112, 343-368.

Kastner-Klein, P. and Rotach, M. W. (2004) Mean Flow and Turbulence Characteristics in an Urban Roughness Sublayer. Boundary-Layer Meteorology, 111, 55. 
Katul, G., Geron, C., Hsieh, C., Vidakovic, B. and Guenther, A. (1998) Active turbulence and scalar transport near the forestatmosphere interface. Journal of Applied Meteorology, 37, 1533-1546.

Katul, G., Hsieh, C.-I., Oren, R., Ellsworth, D. and Phillips, N. (1996) Latent and sensible heat flux predictions from a uniform pine forest using surface renewal and flux variance methods. Boundary-Layer Meteorology, 80, 249-282.

Katul, G. and Liu, H. (2017) A kolmogorov-brutsaert structure function model for evaporation into a turbulent atmosphere. Water Resources Research, 53, 3635-3644.

Katul, G., Porporato, A., Cava, D. and Siqueira, M. (2006) An analysis of intermittency, scaling, and surface renewal in atmospheric surface layer turbulence. Physica D: Nonlinear Phenomena, 215, 117-126.

Kent, C. W., Grimmond, C., Barlow, J., Gatey, D., Kotthaus, S., Lindberg, F. and Halios, C. H. (2017) Evaluation of Urban LocalScale Aerodynamic Parameters: Implications for the Vertical Profile of Wind Speed and for Source Areas. Boundary-Layer Meteorology, 164, 1-31.

Leonardi, S. and Castro, I. P. (2010) Channel flow over large cube roughness: a direct numerical simulation study. Journal of Fluid Mechanics, 651, 519.

Leonardi, S., Orlandi, P., Djenidi, L. and Antonia, R. A. (2015) Heat transfer in a turbulent channel flow with square bars or circular rods on one wall. Journal of Fluid Mechanics, 776, 512-530.

Lhomme, J.-P. (1991) The concept of canopy resistance: historical survey and comparison of different approaches. Agricultural and forest Meteorology, 54, 227-240.

Li, D. and Bou-Zeid, E. (2014) Quality and sensitivity of high-resolution numerical simulation of urban heat islands. Environmental Research Letters, 9, 055001.

Li, D., Rigden, A., Salvucci, G. and Liu, H. (2017) Reconciling the Reynolds number dependence of scalar roughness length and laminar resistance. Geophysical Research Letters, 44, 3193-3200.

Li, Q. and Bou-Zeid, E. (2019) Contrasts between momentum and scalar transport over very rough surfaces. Journal of Fluid Mechanics, 880, 32-58.

Li, Q., Bou-Zeid, E. and Anderson, W. (2016a) The impact and treatment of the Gibbs phenomenon in immersed boundary method simulations of momentum and scalar transport. Journal of Computational Physics, 310, 237-251.

Li, Q., Bou-Zeid, E., Anderson, W., Grimmond, C. and Hultmark, M. (2016b) Quality and reliability of les of convective scalar transfer at high reynolds numbers. International Journal of Heat and Mass Transfer, 102, 959-970.

Li, Q., Bou-Zeid, E., Vercauteren, N. and Parlange, M. B. (2018) Signatures of air-wave interactions over a large lake. BoundaryLayer Meteorology, 167, 445-468.

Llaguno-Munitxa, M. and Bou-Zeid, E. (2018) Shaping buildings to promote street ventilation: A large-eddy simulation study. Urban climate, 26, 76-94.

Macdonald, R. W., Griffiths, R. F. and Hall, D. J. (1998) An improved method for the estimation of surface roughness of obstacle arrays. Atmospheric Environment, 32, 1857-1864.

Masson, V. (2000) A physically-based scheme for the urban energy budget in atmospheric models. Boundary-Layer Meteorology, 94, 357-397.

Monin, A. S. and Obukhov, A. M. (1954) Osnovnye zakonomernosti turbulentnogo peremesivanija v prizemnom sloe atmosfery. Trudy Geofiz. Inst. AN SSSR, 24, 163-187.

Moog, D. and Jirka, G. (1999) Air-water gas transfer in uniform channel flow. Journal of Hydraulic Engineering, 125, 3-10. 
Moriwaki, R. and Kanda, M. (2006a) Scalar Roughness Parameters for a Suburban Area. Journal of the Meteorological Society of Japan, 84, 1063.

- (2006b) Scalar roughness parameters for a suburban area. Journal of the Meteorological Society of Japan. Ser. II, 84, 10631071.

Morrison, W., Yin, T., Lauret, N., Guilleux, J., Kotthaus, S., Gastellu-Etchegorry, J.-P., Norford, L. and Grimmond, S. (2020) Atmospheric and emissivity corrections for ground-based thermography using $3 \mathrm{~d}$ radiative transfer modelling. Remote Sensing of Environment, 237, 111524.

Nikuradse, J. (1933) Laws of Flow in Rough Pipes - Translation of "Stromungsgesetze in rauhen Rohren". Tech. rep.

Owen, P. R. and Thomson, W. (1963) Heat transfer across rough surfaces. Journal of Fluid Mechanics, 15, 321-334.

Perry, A. E., Schofield, W. H. and Joubert, P. N. (1969) Rough wall turbulent boundary layers. Journal of Fluid Mechanics, 37, 383-413.

Placidi, M. and Ganapathisubramani, B. (2015) Effects of frontal and plan solidities on aerodynamic parameters and the roughness sublayer in turbulent boundary layers. Journal of Fluid Mechanics, 782, 541-566.

Poggi, D., Porporato, A., Ridolfi, L., Albertson, J. and Katul, G. (2004) The effect of vegetation density on canopy sub-layer turbulence. Boundary-Layer Meteorology, 111, 565-587.

Pope, S. B. (2000) Turbulent flows. Cambridge: Cambridge University Press.

Raupach, M. R., Finnigan, J. J. and Brunei, Y. (1996) Coherent eddies and turbulence in vegetation canopies: The mixing-layer analogy. Boundary-Layer Meteorology, 78, 351-382.

Ryu, Y. H., Baik, J. J. and Lee, S. H. (2011) A new single-layer urban canopy model for use in mesoscale atmospheric models. Journal of Applied Meteorology and Climatology, 46, 1067-1079.

Schmugge, T., Kustas, W., Ritchie, J., Jackson, T. and Rango, A. (2002) Remote sensing in hydrology. Advances in Water Resources, 25, 1367-1385.

Siqueira, M. B. and Katul, G. G. (2010) An analytical model for the distribution of co 2 sources and sinks, fluxes, and mean concentration within the roughness sub-layer. Boundary-Layer Meteorology, 135, 31-50.

Slotnick, J., Khodadoust, A., Alonso, J., Darmofal, D., Gropp, W., Lurie, E. and Mavriplis, D. (2014) Cfd vision 2030 study: a path to revolutionary computational aerosciences. NASA Tech. Rep. CR-2014-218178, Langley Res. Cent., Hampton, VA.

Snyder, R., Spano, D. and Pawu, K. (1996) Surface renewal analysis for sensible and latent heat flux density. Boundary-Layer Meteorology, 77, 249-266.

Sullivan, P. P., Banner, M. L., Morison, R. P. and Peirson, W. L. (2017) Turbulent Flow over Steep Steady and Unsteady Waves under Strong Wind Forcing. Journal of Physical Oceanography, 48, 3-27.

Theeuwes, N. E., Ronda, R. J., Harman, I. N., Christen, A. and Grimmond, C. S. B. (2019) Parametrizing horizontally-averaged wind and temperature profiles in the urban roughness sublayer. Boundary-Layer Meteorology, 173, 321-348.

Tseng, Y. H., Meneveau, C. and Parlange, M. B. (2006) Modeling flow around bluff bodies and predicting urban dispersion using large eddy simulation. Environmental Science \& Technology, 40, 2653-2662.

Voogt, J. A. and Grimmond, C. S. B. (2000) Modeling Surface Sensible Heat Flux Using Surface Radiative Temperatures in a Simple Urban Area. Journal of Applied Meteorology, 39, 1679.

Wang, Z.-H., Bou-Zeid, E. and Smith, J. A. (2013) A coupled energy transport and hydrological model for urban canopies evaluated using a wireless sensor network. Quarterly Journal of the Royal Meteorological Society, 139, 1643-1657. 
681

Yang, X., Hu, H., Huang, X. and Ge, M. (2019) Drag forces on sparsely packed cube arrays. Journal of Fluid Mechanics.

Yang, X. I. and Abkar, M. (2018) A hierarchical random additive model for passive scalars in wall-bounded flows at high Reynolds numbers. Journal of Fluid Mechanics.

Yang, X. I. A., Sadique, J., Mittal, R. and Meneveau, C. (2016) Exponential roughness layer and analytical model for turbulent boundary layer flow over rectangular-prism roughness elements. Journal of Fluid Mechanics, 789, 127.

Zhu, X. and Anderson, W. (2018) Turbulent flow over urban-like fractals: prognostic roughness model for unresolved generations. Journal of Turbulence, 19, 995-1016.

Zhu, X., lungo, G. V., Leonardi, S. and Anderson, W. (2017) Parametric study of urban-like topographic statistical moments relevant to a priori modelling of bulk aerodynamic parameters. Boundary-Layer Meteorology, 162, 231-253.

Zilitinkevich, S. S., Grachev, A. A. and Fairall, C. W. (2001) Notes and correspondece: Scaling reasoning and field data on the sea surface roughness lengths for scalars. Journal of the Atmospheric Sciences, 58, 320-325. 